\title{
Physicians Voluntarily Using an EHR-Based CDS Tool Improved Patients' Guideline-Related Statin Prescription Rates: A Retrospective Cohort Study
}

\author{
Timothy S. Chang ${ }^{1}$ Ashwin Buchipudi ${ }^{2}$ Gregg C. Fonarow ${ }^{3}$ Michael A. Pfeffer ${ }^{4}$ Jennifer S. Singer ${ }^{5}$ \\ Eric M. Cheng ${ }^{1}$
}

${ }^{1}$ Department of Neurology, University of California, Los Angeles, Los Angeles, California, United States

2 Information Services and Solutions, University of California, Los Angeles, Los Angeles, California, United States

${ }^{3}$ Division of Cardiology, Department of Medicine, University of California, Los Angeles, Los Angeles, California, United States

${ }^{4}$ Division of General Internal Medicine, Department of Medicine, University of California, Los Angeles, Los Angeles, California, United States

${ }^{5}$ Department of Urology, University of California, Los Angeles, Los Angeles, California, United States

\author{
Address for correspondence Timothy S. Chang, MD, PhD, \\ Department of Neurology, University of California, Los Angeles, \\ 695 Charles E Young Dr South, Room 2309, Los Angeles, CA 90095 , \\ United States (e-mail: timothychang@mednet.ucla.edu).
}

Appl Clin Inform 2019;10:421-445.

\section{Abstract}

Keywords
- electronic health
records
- decision support
techniques
- anticholesteremic
agents
- atherosclerosis

Background In 2013, the American College of Cardiology (ACC) and the American Heart Association (AHA) released a revised guideline on statin therapy initiation. The guideline included a 10-year risk calculation based on regression modeling, which made hand calculation infeasible. Compliance to the guideline has been suboptimal, as many patients were recommended but not prescribed statin therapy. Clinical decision support (CDS) tools may improve statin guideline compliance. Few statin guideline CDS tools evaluated clinical outcome.

Objectives We determined if use of a CDS tool, the statin macro, was associated with increased 2013 ACC/AHA statin guideline compliance at the level of statin prescription versus no statin prescription. We did not determine if each patient's statin prescription met ACC/AHA 2013 therapy intensity recommendations (high vs. moderate vs. low). Methods The authors developed a clinician-initiated, EHR-embedded statin macro command ("statin macro") that displayed the 2013 ACC/AHA statin guideline recommendation in the electronic health record documentation. We included patients who had a primary care visit during the study period (January 1-June 30, 2016), were eligible for statin therapy based on the ACC/AHA guideline prior to the study period, and were not prescribed statin therapy prior to the study period. We tested the association of macro usage and statin therapy prescription during the study period using relative risk and mixed effect logistic regression.

Results Subjects included 11,877 patients seen in primary care, who were retrospectively recommended statin therapy at study initiation based on the ACC/AHA guideline, but who had not received statin therapy. During the study period, 125 received

January 2, 2019

accepted after revision

April 26, 2019 (c) 2019 Georg Thieme Verlag KG Stuttgart . New York
DOI https://doi.org/

10.1055/s-0039-1692186. ISSN 1869-0327. 
clinicians used the statin macro command for 389 of the 11,877 patients (3.2\%). Of the 389 patients for whom that statin macro was used, 108 patients $(28 \%)$ had a statin prescribed during the study period. Of the 11,488 for whom the statin macro was not used, 1,360 (13\%) patients received a clinician-prescribed statin (relative risk 2.3, $p<0.001$ ). Controlling for patient covariates and clinicians, statin macro usage was significantly associated with statin therapy prescription (odds ratio 2.86, $p<0.001$ ). Conclusion Although the statin macro had low uptake, its use was associated with a greater rate of statin prescriptions (dosage not determined) for patients whom 2013 ACC/AHA guidelines required statin therapy.

\section{Background and Significance}

Although many best practice guidelines exist for initiating medication in select patient groups, clinicians prescribe the targeted medications at suboptimal rates. ${ }^{1}$ In 2013, the American College of Cardiology (ACC) and the American Heart Association (AHA) released a revised guideline regarding statin therapy initiation ${ }^{2}$ (see - Fig. 1 and - Supplementary Material (available in the online version) for a brief overview of the 2013 ACC/AHA statin guideline). Both the 2013 ACC/AHA statin guideline compliance, and the percentage of patients prescribed statin therapy per the guideline, varied by patient population. In 2015, cardiologists prescribed 2013 guidelinecompliant, secondary prevention statin therapy to $91 \%$ of their patients with atherosclerotic cardiovascular disease (ASCVD). ${ }^{3}$ However, prevention guideline compliance was suboptimal in primary care patients. In the 4 years following 2013 ACC/AHA statin guideline release, $42 \%$ of patients with no history of ASCVD and a 10-year ASCVD risk score $>7.5 \%$ received statin prescription in accordance with the ACC/AHA statin guideline. $^{4}$

A postulated reason for suboptimal 2013 ACC/AHA statin guideline compliance was its complexity. ${ }^{5,6}$ While previous statin guidelines utilized integer-based risk scores that could be determined by hand, ${ }^{7}$ the 2013 ACC/AHA statin guideline utilized four regression-based risk scoring equations that required use of a calculator. Which 2013 ACC/AHA regression equation to use depended on a patient's gender and race. Each regression equation incorporated age, high-density lipoprotein (HDL), total cholesterol, diabetes mellitus (DM) history, systolic blood pressure, antihypertensive medication, and smoking status. The 10-year ASCVD risk calculation determined if statin therapy should be initiated in patients without DM and with low-density lipoprotein (LDL) values between 70 and $189 \mathrm{mg} / \mathrm{dL}$. Although other existing 10-year risk calculators such as Framingham ${ }^{8}$ and QRISK $^{9}$ were available, the ACC/AHA developed their 2013 new 10-year risk calculator based on regression equations. Concurrent with the 2013 ACC/AHA statin guideline publication, the ACC/AHA released mobile and online ASCVD Risk Estimator calculators. ${ }^{10,11}$ However, clinicians had to manually enter information, which was time consuming. ${ }^{12}$ These calculators were available prior to the studies showing suboptimal 2013 ACC/AHA statin guideline compliance. ${ }^{4}$ The current study authors hypothesized that a clinical decision support (CDS) tool that automatically retrieved patient data and performed regression calculation could improve 2013 ACC/AHA statin guideline compliance.

Previous CDS tools improved guideline compliance with modest benefit ${ }^{13}$ for $\mathrm{DM},{ }^{14}$ heart failure, ${ }^{15}$ and pneumonia. ${ }^{16}$ Childhood vaccination rates improved when implemented within electronic health record (EHR) templates, with preloaded immunization records, and alerts. ${ }^{17}$ In randomized control trials, CDS tools with features such as automation, on-screen display, system initiation, and advice to patients as well as clinicians were more effective. Features such as advise within charting or order entry were less effective. ${ }^{18,19}$

A systematic review identified 34 previous health care intervention tools tested in randomized control trials for lipid management. ${ }^{20}$ Five CDS tools were integrated into an EHR. For example, the MayoExpertAdvisor study, based on a Web service with prefilled patient data from the EHR, provided 2013 ACC/AHA ASCVD risk calculation. That study showed decreased time for clinicians to determine a statin therapy recommendation, but did not report whether guideline compliance improved. ${ }^{21}$ In a systematic review, a low percentage of CDS tools evaluated clinical benefit. ${ }^{22}$

Within the authors' EHR system, Epic, ${ }^{23}$ clinicians can initiate CDS tools during note documentation. Such tools can automatically retrieve patient data and perform calculations. Therefore, study clinicians did not need to exit the EHR to perform calculations on an external platform. Previous macro commands were developed at other institutions for obesity counseling ${ }^{24}$ and H1N1 swine flu recommendations. ${ }^{25}$ However, those macros did not retrieve patient data or perform calculations.

We implemented the study's statin macro in July 2014, shortly after the November 2013 ACC/AHA guideline and online calculator publications. Unlike earlier statin CDS tools elsewhere that were not integrated into the EHR, ${ }^{21}$ the current study's statin macro was accessible during EHR note generation. The previous tools had been printed on paper forms, ${ }^{26,27}$ shown on a separate screen, ${ }^{14,28}$ or emailed to clinicians. ${ }^{29}$

\section{Objectives}

We determined if the statin macro was associated with statin prescription, regardless of dose, per the 2013 ACC/AHA statin 


\section{ACC/AHA STATIN GUIDELINES}

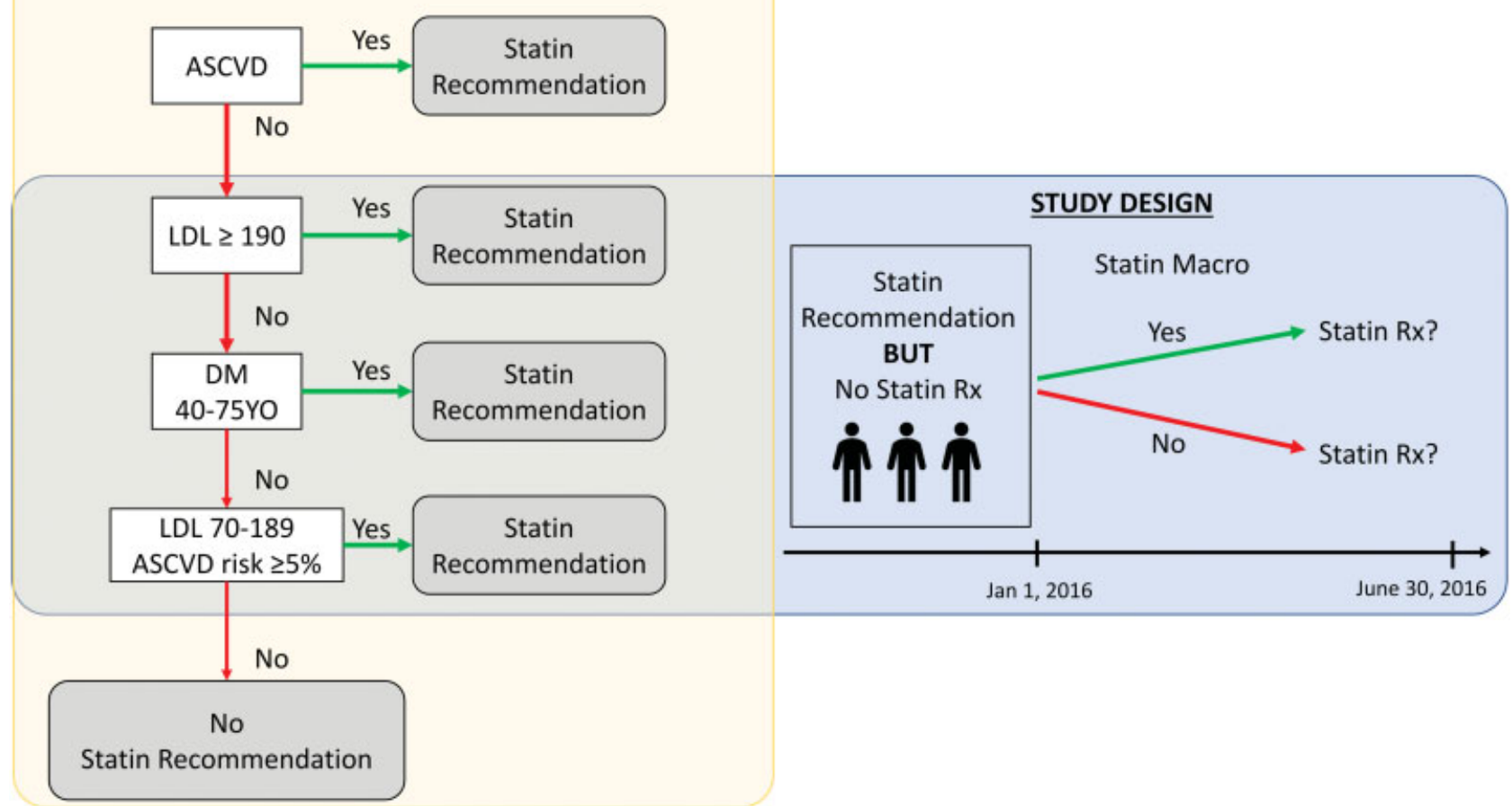

Fig. 1 Central schematic including simplified version of the 2013 American College of Cardiology/American Heart Association (ACC/AHA) statin guideline and study design. For the statin guidelines, patients with a history of ASCVD, LDL $\geq 190 \mathrm{mg} / \mathrm{dL}$, or DM aged 40 to 75 years old were recommended statin therapy. Patients without DM aged 40 to 75 years old and an LDL of 70 to $189 \mathrm{mg} / \mathrm{dL}$ necessitated 10-year ASCVD risk calculation. Patients with a 10-year ASCVD risk calculation $\geq 5 \%$ were recommended statin therapy while those with a 10-year ASCVD risk calculation $<5 \%$ were not recommended statin therapy. For the study design, patients recommended statin therapy for primary prevention (LDL $\geq 190 \mathrm{mg} / \mathrm{dL}$; DM and 40-75 years old; no DM, LDL 70-189 mg/dL and 10-year ASCVD risk $\geq 5 \%$ ) but not prescribed statin therapy as of December 31, 2015, were included in this study. We tested the association of macro usage and statin therapy prescription during the study period (January 1, 2016-June 30, 2016). Abbreviations: ASCVD, atherosclerotic cardiovascular disease; ASCVD risk, 10-year ASCVD risk calculation; DM, diabetes mellitus; LDL, low-density lipoprotein; Rx, prescription; YO, years old.

guidelines. Specifically, for patients retrospectively recommended (at the time of study initiation) but not prescribed statin therapy, we investigated whether subsequent statin macro usage was associated with statin prescription. We did not determine if each patient's statin prescription met the ACC/AHA 2013 therapy intensity recommendations (high vs. moderate vs. low). - Fig. 1 shows a central schematic of the study design.

\section{Methods}

\section{Statin Macro Development}

After the November 2013 ACC/AHA statin guideline was published, and prior to the statin macro implementation, we received multiple requests from clinicians to provide a CDS tool for the ACC/AHA statin guideline. We did not assess clinician knowledge of or compliance with the 2013 ACC/ AHA statin guideline in our health system. We hypothesized a statin macro could improve knowledge about the 2013 ACC/AHA statin guideline and also guideline compliance.

A multidisciplinary expert panel including cardiologists, internists, neurologists, clinical informaticians, and quality officers developed the statin macro, which was based on the published ACC/AHA statin guideline. Developers tested hundreds of patients for accuracy of the macro's statin guideline recommendations. During statin macro development, expert panel members revised it several times prior to the study. Revisions led to statin therapy recommendations based on 17 scenarios of a patient's ASCVD history, DM history, LDL level, 10-year ASCVD risk, and medication list (- Supplementary Table S1, available in the online version). For example, the macro suppressed a statin recommendation if the patient had a statin allergy. Multiple governance committees, including the institutions' primary care leadership, approved use of the macro. The study institution's Institutional Review Board (IRB) approved a waiver of authorization for this study (IRB\#: 16-001676).

The clinician-initiated CDS tool was relevant and manageable. The statin macro made a clear recommendation of statin therapy or no statin therapy. The statin macro contained a hyperlink to the 2013 ACC/AHA statin guideline and the ACC/AHA ASCVD Risk Estimator online calculator. ${ }^{10}$ The ASCVD Risk Estimator hyperlink allowed clinicians to manually confirm macro recommendations. Errors found in the statin macro could be logged as a ticket to the EHR help desk.

In the study site's Epic EHR terminology, the CDS tool we developed is known as a Smart Phrase. Authors refer to the Smart Phrase herein by its generic name, "macro command," to avoid vendor-specific terminology. While documenting a note, clinicians could type an abbreviated phrase such as "CVRISK" 
to invoke the statin macro directly and incorporate its output into the note. Macros of this sort are clinician-initiated CDS tools that are "pulled" by clinicians. Clinician-initiated CDS tools contrast with system-initiated CDS tools that are automatically "pushed" to clinicians as alerts. -Fig. 2 shows a screenshot of the statin macro within a note. Development of the statin macro was consistent with the GUIDES checklist. ${ }^{30}$ The statin macro automatically retrieved patient data from the EHR. Developers tested hundreds of patients for accuracy of patient data retrieval. In addition to activating the statin macro directly while documenting a clinical note, clinicians could alternatively add the macro to a note template, allowing the macro to upload with every use of the encompassing template.

While EHRs have high consistency, they do not have 100\% completeness or correctness. ${ }^{31,32}$ The physician-targeted statin macro delivered consistent, on demand, and fast recommendations within the clinicians' note documentation workflow. Statin macro versions included those that showed a summary and those that showed all variable values (-Table 1). Variables were highlighted in blue. Clinicians could customize CDS delivery with these variations.

\section{Statin Macro Implementation}

The study institution installed an enterprise-wide version of the EHR from Epic ${ }^{23}$ in over 170 urban clinics and 3 hospitals at a large, urban academic medical center from March 2013 to July 2014. Epic was installed in all primary care clinics by July 11, 2013. The statin macro was first accessible to clinicians on July 21,2014 . January 1,2016 to June 30, 2016 defined the study period.

One of the study sites' biweekly ambulatory EHR email updates described the statin macro and encouraged usage. Clinician awareness of the statin macro also spread through word of mouth. As a clinician-initiated CDS tool, clinicians using the macro were likely motivated and believed its usage would improve patient care. The study did not measure the study clinicians' assessment of their premacro-usage likelihood of prescribing a statin for the patient at hand.

The email advertising the statin macro included instructions. Prior to statin macro implementation, we did not assess factors that would influence guideline compliance. Because the statin macro was a clinician-initiated CDS tool, clinicians were not forced to follow recommendations or explain why they did not follow recommendations. The institution's clinical leadership supported the statin macro but did not provide incentives for statin guideline compliance.

\section{Study Criteria}

January 1, 2016 to June 30, 2016 defined the study period. We chose this 6-month period based on prestudy estimates for the number of times clinicians used the statin macro. As a retrospective cohort study, clinicians did not know during the study period they would be included in this study. Inclusion criteria were patients:

- Who had a primary care visit during the study period. Any outpatient visit with a patient's primary care clinician defined a primary care visit.

- Who were 40 to 75 years old as of January 1, 2016. The 10year ASCVD risk calculator was developed for this age range.

- Who did not have a statin prescription before the study period as of December 31, 2015.

- Who were retrospectively identified as candidates for statin therapy based on the 2013 ACC/AHA statin guideline before the study period as of December 31, 2015.

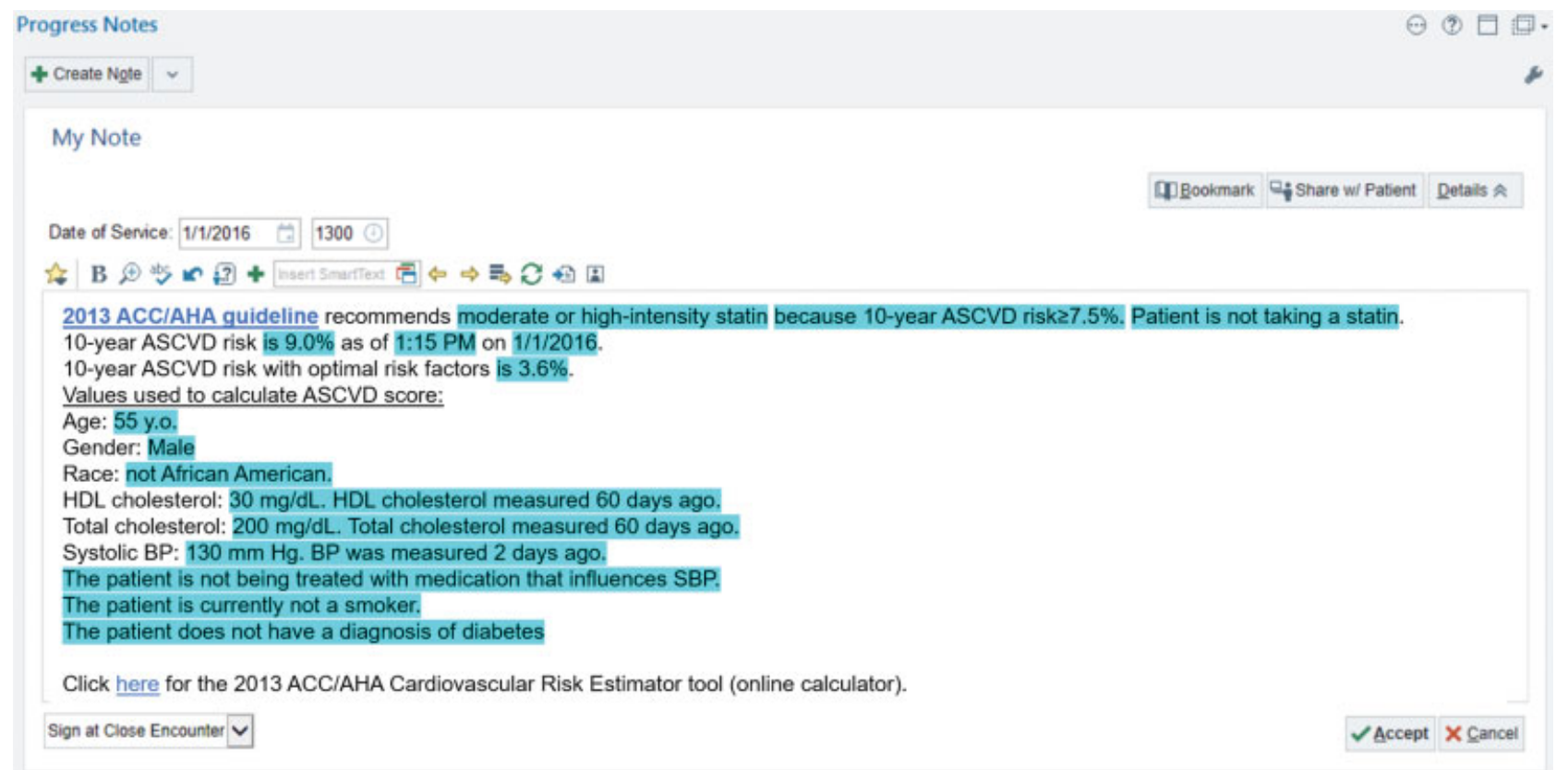

Fig. 2 Screenshot of the statin macro within a note. Blue highlighted text was variable and specific to each patient. The blue "2013 American College of Cardiology/American Heart Association (ACC/AHA) guideline" text included a hyperlink to the 2013 ACC/AHA statin guideline. The blue "here" text included a hyperlink to the online ASCVD Risk Estimator calculator (Screenshot used with permission from @ 2019 Epic Systems Corporation). 


\begin{tabular}{|c|c|}
\hline $\begin{array}{l}\text { Version 1: Recommendation } \\
\text { Hyperlinked } 2013 \text { ACC/AHA guideline followed by } \\
\text { recommendation and statin status }\end{array}$ & $\begin{array}{l}2013 \text { ACC/AHA guideline recommends moderate or high-intensity statin } \\
\text { because } 10 \text {-year ASCVD risk } \geq 7.5 \% \text {. Patient is not taking a statin }{ }^{a}\end{array}$ \\
\hline $\begin{array}{l}\text { Version 2: Brief } \\
\text { Hyperlinked } 2013 \text { ACC/AHA guideline followed by } \\
\text { recommendation and statin status } \\
\text { The second line shows the risk calculator results if it } \\
\text { should be calculated }\end{array}$ & $\begin{array}{l}2013 \text { ACC/AHA guideline recommends moderate or high-intensity statin } \\
\text { because } 10 \text {-year ASCVD risk } \geq 7.5 \% \text {. Patient is not taking a statin. } \\
\text { Ten-year ASCVD risk is } 9.0 \% \text { as of } 1: 15 \text { PM on January } 1,2016\end{array}$ \\
\hline $\begin{array}{l}\text { Version 3: Full } \\
\text { Hyperlinked } 2013 \text { ACC/AHA guideline followed by } \\
\text { recommendation and statin status } \\
\text { The second line shows the risk calculator results if it } \\
\text { should be calculated } \\
\text { The third line shows the optimal risk score } \\
\text { The following lines show the values used to calculate } \\
\text { ASCVD score }\end{array}$ & $\begin{array}{l}2013 \text { ACC/AHA guideline recommends moderate or high-intensity statin } \\
\text { because } 10 \text {-year ASCVD risk } \geq 7.5 \% \text {. Patient is not taking a statin } \\
\text { Ten-year ASCVD risk is } 9.0 \% \text { as of } 1: 15 \text { PM on January } 1,2016 \\
\text { Ten-year ASCVD risk with optimal risk factors is 3.6\% } \\
\text { Values used to calculate ASCVD score: } \\
\text { Age: } 55 \text { years old } \\
\text { Gender: Male } \\
\text { Race: not African American } \\
\text { HDL cholesterol: } 30 \mathrm{mg} / \mathrm{dL} \text {. HDL cholesterol measured } 60 \text { days ago } \\
\text { Total cholesterol: } 200 \mathrm{mg} / \mathrm{dL} \text {. Total cholesterol measured } 60 \text { days ago } \\
\text { Systolic BP: } 130 \mathrm{~mm} \mathrm{Hg} \text {. BP was measured } 2 \text { days ago } \\
\text { The patient is not being treated with medication that influences SBP } \\
\text { The patient is currently not a smoker } \\
\text { The patient does not have a diagnosis of diabetes } \\
\text { Click here for the } 2013 \mathrm{ACC} / \mathrm{AHA} \text { Cardiovascular Risk Estimator tool } \\
\text { (online calculator) }\end{array}$ \\
\hline
\end{tabular}

Abbreviations: ACC, American College of Cardiology; AHA, American Heart Association; ASCVD, atherosclerotic cardiovascular disease; BP, blood pressure; HDL, high-density lipoprotein; SBP, systolic blood pressure. ablue highlighted text was variable and specific to each patient.

Exclusion criteria were patients:

- Who had a statin allergy.

- Who had a history of ASCVD. Patients with a history of ASCVD were recommended statin therapy based on the 2013 ACC/AHA statin guideline for secondary prevention.

- Who did have a statin prescription before the study period as of December 31, 2015.

- Who did not have sufficient data to determine the 2013 ACC/AHA statin guideline recommendation. For example, some patients did not have a LDL measurement or were missing data necessary to calculate the 10-year ASCVD risk (e.g., blood pressure measurement).

- Who were not recommended statin therapy based on the 2013 ACC/AHA statin guideline before the study period as of December 31, 2015.

We extracted EHR data as of December 31, 2015, necessary to determine the ACC/AHA statin guideline recommendation. Data included the patients' age, gender, smoking status, visit diagnoses, and problem list. We extracted the most recent data prior to December 31, 2015, for blood pressure (looking back to January 1, 2014) and cholesterol (total, low-density, and high-density: looking back to January 1,2011$)$. We extracted statin and antihypertensive medication (December 31, 2015-June 30, 2016, including start and end date of medications), allergies (as of June 30, 2016), and statin macro usage (January 1, 2016-June 30, 2016). Searching note text for "2013 ACC/AHA guideline*10year ASCVD risk," where * indicated additional text that may be between these phrases, identified statin macro usage. All statin macro versions had these phrases.

\section{Exposure and Outcome}

Any version of statin macro usage during the study period defined the exposure. Statin therapy prescription of any dosage during the study period defined the outcome. A statin prescription with a start date from January 1, 2016, to June 30, 2016, or an end date after June 30, 2016, defined statin prescription during the study period. Prescriptions that were hand written or called into a pharmacy, and not entered in the EHR, were not accounted for.

\section{Analyses}

For patients meeting the inclusion/exclusion criteria, we compared variables between patients for whom the statin macro was and was not used. The Student's t-test was used for continuous variables (age, systolic blood pressure, total cholesterol, LDL, HDL) and the binomial test was used for binary variables (gender, race, smoking, DM, antihypertensive medication). For clinicians who did and did not use the statin macro, we compared clinician type, gender, and specialty using the binomial test.

We calculated the relative risk of statin therapy prescription for macro usage compared with no macro usage. To control for patient covariates and clinicians, we used a mixed effect logistic regression model. The response variable was statin therapy prescription. The dependent variables included macro usage and variables used for the ACC/AHA statin guideline recommendation. As multiple patients may have been seen by the same primary care clinician, we included patients' clinician as a random effect, where each clinician had a specific random intercept. We used the likelihood ratio test to determine the significance of variables in 


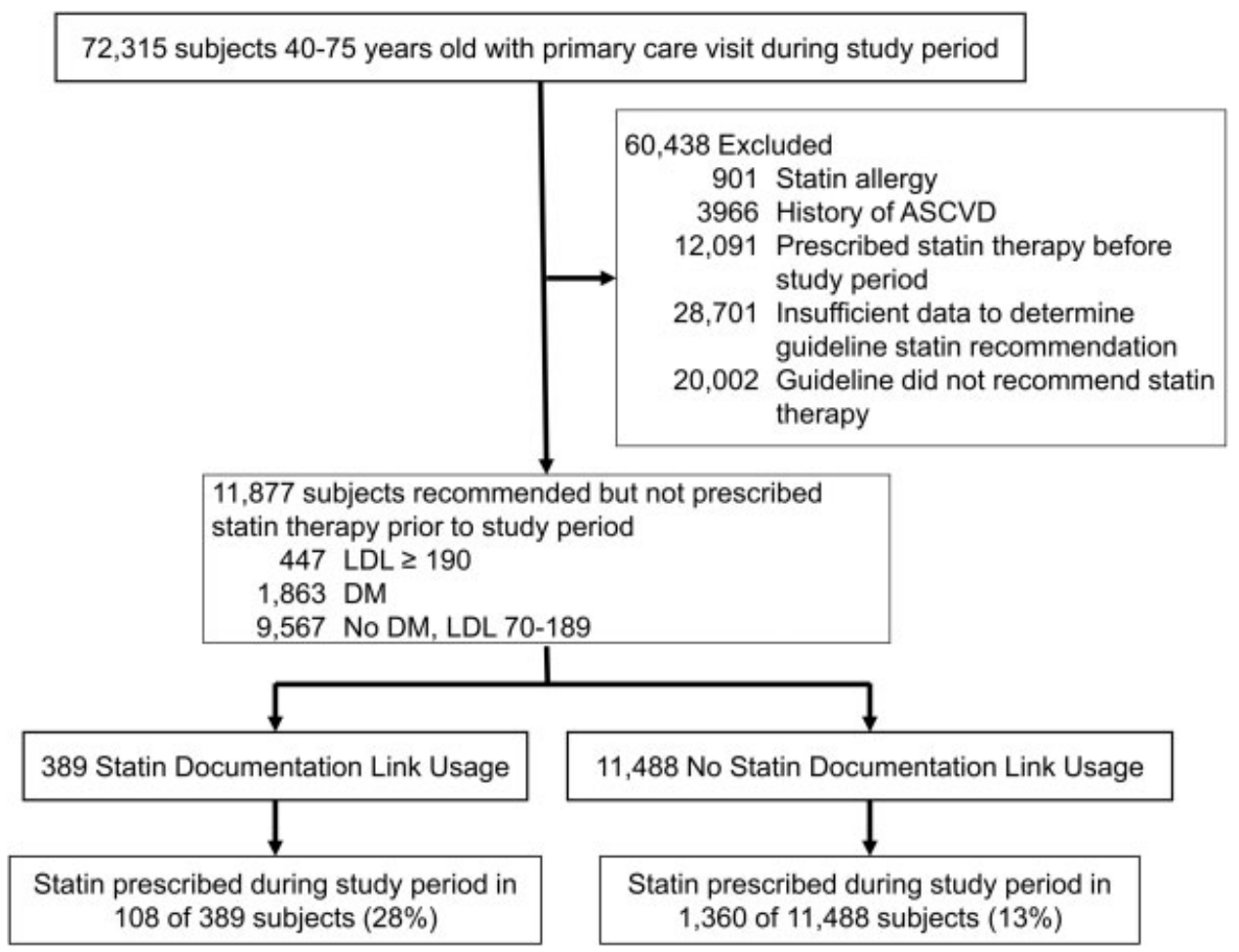

Fig. 3 Flow diagram showing study exclusion criteria, inclusion criteria, and results. 72,315 patients aged 40 to 75 years had a primary care visit during the study period. A total of 60,438 patients were excluded. Counts for categories of excluded patients are shown. 11,877 patients were recommended statin therapy based on the 2013 American College of Cardiology/American Heart Association (ACC/AHA) statin guideline but not prescribed statin therapy prior to the study period. Counts for categories of included patients are shown. Statin therapy was prescribed during the study period in $28 \%$ (108 of 389 ) of patients for whom the statin macro was used compared with $13 \%(1,360$ of 11,488$)$ of patients for whom the statin macro was not used. Abbreviations: ASCVD, atherosclerotic cardiovascular disease; DM, diabetes mellitus; LDL, low-density lipoprotein.

the model. Wald confidence intervals were calculated for odds ratios of fixed effect variables in the model. ${ }^{33}$

In this primary analysis, patients without complete data were removed. Because missing data precluded statin recommendation for some patients, we repeated analysis on the final imputed data set of a multiple imputation procedure with predictive mean matching ${ }^{34-36}$ using the Hmisc package. ${ }^{37}$ See - Supplementary Material (available in the online version) for further details on missing data analysis. All analyses were performed in R (version 3.5.2). ${ }^{38}$

\section{Results}

From January 1, 2016, to June 30, 2016, 72,315 patients aged 40 to 75 years had a primary care visit. Of those, 60,438 patients were dropped based on the study exclusion criteria (-Fig. 3). Thus, 11,877 study eligible patients met the inclusion/exclusion criteria. - Table 2 shows baseline characteristics of patients included in the study. DM was significantly less common in the statin macro usage group (11\% vs. $16 \%$, $p<0.05)$. In contrast, LDL levels were higher in the macro usage group (127 vs. $123 \mathrm{mg} / \mathrm{dL}, p<0.05$ ).

A total of 443 primary care clinicians cared for study eligible patients. Of those, 440 clinicians did not use the statin macro on at least one patient while 125 clinicians used the statin macro on at least one patient. Because they used the statin macro for some patients and did not use the statin macro for other patients, 122 clinicians were in both groups. -Table 3 compares type, gender, and specialty of clinicians who did and did not use the statin macro. Most clinicians were internal medicine and family medicine physicians. There were significantly more residents who did not use the statin macro (5\%) compared with those who used the statin macro (1\%).

For each clinician who used the statin macro at least once in study eligible or ineligible patients, -Table 4 shows the total number of patients seen, statin macro usage, and statin prescription stratified by patient study eligibility. The number of study eligible patients seen per clinician who used the statin macro at least once ranged from 1 to 284 . For study eligible patients, the top 33 clinician statin macro users (of 125 physicians) contributed $62 \%$ of all macro usages. For each clinician who never used the statin macro and prescribed statin therapy at least once, - Table 5 shows the number of patients seen and statin prescription in study eligible and ineligible patients. The number of study eligible patients seen per clinician who never used the statin macro and prescribed statin therapy at least once ranged from 1 to 197.

For study eligible patients, the statin macro was used in $3.2 \%$ (389 of 11,877 ) of patients and not used in 11,488 patients. Clinicians prescribed statin therapy during the study period in $28 \%$ (108 of 389 ) of patients for whom the statin macro was used compared with $13 \%(1,360$ of 11,488$)$ of patients for whom the statin macro was not used (-Fig. 3 ). 
Table 2 Baseline characteristics of patients meeting the inclusion/exclusion criteria stratified by statin macro usage $(N=11,877)$

\begin{tabular}{|l|l|l|l|}
\hline & $\begin{array}{l}\text { Statin macro usage } \\
(N=389)\end{array}$ & $\begin{array}{l}\text { No statin macro usage } \\
(N=11,488)\end{array}$ & $p$-Value \\
\hline Male & $55 \%(213)$ & $55 \%(6,322)$ & 0.96 \\
\hline Age $(\mathrm{y})$ & $62.8(8.1)$ & $63.3(7.7)$ & 0.23 \\
\hline Black & $11 \%(44)$ & $8.9 \%(1,019)$ & 0.12 \\
\hline Smoke & $8.2 \%(32)$ & $7.1 \%(810)$ & 0.43 \\
\hline DM & $11 \%(42)$ & $16 \%(1,863)$ & 0.005 \\
\hline Antihypertensive & $39 \%(151)$ & $39 \%(4,510)$ & 0.9 \\
\hline Systolic BP $(\mathrm{mm} \mathrm{Hg})$ & $132(17)$ & $132(17)$ & 0.84 \\
\hline Total cholesterol $(\mathrm{mg} / \mathrm{dL})$ & $210(35)$ & $207(37)$ & 0.15 \\
\hline LDL $(\mathrm{mg} / \mathrm{dL})$ & $127(29)$ & $123(31)$ & 0.006 \\
\hline HDL $(\mathrm{mg} / \mathrm{dL})$ & $57(16)$ & $59(19)$ & 0.13 \\
\hline
\end{tabular}

Abbreviations: BP, blood pressure; DM, diabetes mellitus; HDL, high-density lipoprotein; LDL, low-density lipoprotein.

Note: Numbers in parenthesis correspond to total patients or standard deviation for binary and continuous variables, respectively.

Table 3 Characteristics for clinicians of patients who met the inclusion/exclusion criteria stratified by statin macro usage $(N=443)$

\begin{tabular}{|l|l|l|l|}
\hline & $\begin{array}{l}\text { Statin macro usage } \\
\text { on at least 1 patient } \\
(N=125)\end{array}$ & $\begin{array}{l}\text { No statin macro usage } \\
\text { on at least 1 patient } \\
(N=440)\end{array}$ & $p$-Value \\
\hline Type & & & 0.023 \\
\hline Physician & $98 \%(123)$ & $92 \%(406)$ & 0.047 \\
\hline Resident & $1 \%(1)$ & $5 \%(24)$ & 0.82 \\
\hline Fellow & $1 \%(1)$ & $2 \%(7)$ & 0.82 \\
\hline Nurse practitioner & $0 \%(0)$ & $1 \%(3)$ & \\
\hline Sex & & & 0.12 \\
\hline Male & $54 \%(67)$ & $45 \%(199)$ & 0.70 \\
\hline Female & $46 \%(58)$ & $49 \%(215)$ & 0.011 \\
\hline Unknown & $0 \%(0)$ & $6 \%(26)$ & \\
\hline Specialty & & & 0.45 \\
\hline Internal medicine & $66 \%(83)$ & $71 \%(310)$ & 0.06 \\
\hline Family medicine & $34 \%(42)$ & $25 \%(108)$ & 0.34 \\
\hline Surgery & $0 \%(0)$ & $2 \%(7)$ & 0.64 \\
\hline Neurology & $0 \%(0)$ & $1 \%(4)$ & 0.82 \\
\hline Obstetrics and gynecology & $0 \%(0)$ & $2 \%(8)$ & 0.28 \\
\hline Unknown & $0 \%(0)$ & & \\
\hline
\end{tabular}

Note: Numbers in parenthesis correspond to total patients.

The relative risk of statin therapy prescription for macro usage compared with no macro usage was 2.3 (95\% confidence interval $[\mathrm{CI}], 1.9-2.8, p<0.001)$. Statin therapy prescription was significantly more likely in patients for whom the statin macro was used (odds ratio $2.86,95 \% \mathrm{CI}$, 2.24-3.65, $p<0.001$ ) while controlling for gender, age, race, smoking status, ASCVD, DM, systolic blood pressure, antihypertensive medication, total cholesterol, LDL, HDL, and clinician (-Table 6). Clinician was modeled as a random effect and had a significant variance among clinicians (odds ratio $1.36, p<0.001$ ).
We investigated only the clinicians who had evidence of statin macro usage. From - Table 4 , clinicians that used the statin macro at least once in study eligible or ineligible patients saw a total of 9,515 study eligible patients. These clinicians prescribed statin therapy during the study period in $28 \%$ (108 of 389 ) of patients for whom the statin macro was used compared with $12 \%(1,110$ of 9,126$)$ of patients for whom the statin macro was not used. The relative risk of statin therapy prescription for macro usage compared with no macro usage was 2.3 (95\% CI, 1.9-2.7, $p<0.001$ ). Statin therapy prescription was significantly more likely in 
Table 4 For clinicians who used the statin macro at least once, clinician statin macro usage and prescription stratified by patient study eligibility

\begin{tabular}{|c|c|c|c|c|c|c|c|c|}
\hline & Study eligib & e patients & & & Study inelig & ble patient & & \\
\hline $\begin{array}{l}\text { Clinician } \\
\text { ID }\end{array}$ & $\begin{array}{l}\text { Total no. } \\
\text { of patients }\end{array}$ & $\begin{array}{l}\text { No. of } \\
\text { patients } \\
\text { macro } \\
\text { used }\end{array}$ & $\begin{array}{l}\text { No. of } \\
\text { patients } \\
\text { macro } \\
\text { used more } \\
\text { than once }\end{array}$ & $\begin{array}{l}\text { No. of } \\
\text { patients } \\
\text { statin } \\
\text { prescribed } \\
\text { (macro } \\
\text { used, } \\
\text { macro not } \\
\text { used) }\end{array}$ & $\begin{array}{l}\text { Total no. } \\
\text { of patients }\end{array}$ & $\begin{array}{l}\text { No. of } \\
\text { patients } \\
\text { macro } \\
\text { used }\end{array}$ & $\begin{array}{l}\text { No. of } \\
\text { patients } \\
\text { macro } \\
\text { used more } \\
\text { than once }\end{array}$ & $\begin{array}{l}\text { No. of } \\
\text { patients } \\
\text { statin } \\
\text { prescribed } \\
\text { (macro } \\
\text { used, } \\
\text { macro not } \\
\text { used) }\end{array}$ \\
\hline 16419 & 284 & 20 & 0 & $19(5,14)$ & 657 & 19 & 0 & $\begin{array}{l}290 \\
(11,279)\end{array}$ \\
\hline 18983 & 222 & 1 & 1 & $4(1,3)$ & 942 & 3 & 0 & $\begin{array}{l}139 \\
(1,138)\end{array}$ \\
\hline 15692 & 212 & 4 & 0 & $18(3,15)$ & 614 & 2 & 0 & $\begin{array}{l}269 \\
(1,268)\end{array}$ \\
\hline 15026 & 189 & 12 & 2 & $23(7,16)$ & 1,085 & 43 & 0 & $\begin{array}{l}249 \\
(15,234)\end{array}$ \\
\hline 21490 & 173 & 1 & 0 & $11(0,11)$ & 442 & 3 & 0 & $\begin{array}{l}150 \\
(1,149)\end{array}$ \\
\hline 17711 & 167 & 1 & 1 & $12(1,11)$ & 549 & 2 & 0 & $\begin{array}{l}196 \\
(1,195)\end{array}$ \\
\hline 19343 & 165 & 3 & 0 & $20(2,18)$ & 553 & 2 & 0 & $\begin{array}{l}214 \\
(0,214)\end{array}$ \\
\hline 15688 & 153 & 2 & 0 & $13(1,12)$ & 424 & 5 & 0 & $\begin{array}{l}198 \\
(2,196)\end{array}$ \\
\hline 19301 & 140 & 9 & 2 & $5(1,4)$ & 256 & 9 & 2 & $69(0,69)$ \\
\hline 16389 & 139 & 0 & 0 & $17(0,17)$ & 634 & 2 & 0 & $\begin{array}{l}325 \\
(1,324)\end{array}$ \\
\hline 15007 & 132 & 1 & 0 & $11(0,11)$ & 552 & 2 & 0 & $\begin{array}{l}143 \\
(0,143)\end{array}$ \\
\hline 15684 & 131 & 11 & 2 & $20(7,13)$ & 588 & 25 & 3 & $\begin{array}{l}185 \\
(5,180)\end{array}$ \\
\hline 26752 & 131 & 1 & 0 & $24(0,24)$ & 498 & 6 & 0 & $\begin{array}{l}196 \\
(0,196)\end{array}$ \\
\hline 9121 & 130 & 1 & 0 & $4(0,4)$ & 451 & 2 & 0 & $\begin{array}{l}117 \\
(0,117)\end{array}$ \\
\hline 29618 & 127 & 3 & 0 & $8(1,7)$ & 336 & 4 & 0 & $80(0,80)$ \\
\hline 11186 & 126 & 13 & 1 & $8(2,6)$ & 273 & 9 & 0 & $\begin{array}{l}115 \\
(1,114)\end{array}$ \\
\hline 20857 & 116 & 1 & 0 & $13(0,13)$ & 434 & 2 & 0 & $\begin{array}{l}115 \\
(0,115)\end{array}$ \\
\hline 19609 & 110 & 3 & 0 & $9(1,8)$ & 438 & 2 & 1 & $\begin{array}{l}108 \\
(1,107)\end{array}$ \\
\hline 14796 & 109 & 1 & 0 & $13(1,12)$ & 259 & 1 & 0 & $\begin{array}{l}116 \\
(0,116)\end{array}$ \\
\hline 15368 & 102 & 5 & 0 & $10(2,8)$ & 427 & 6 & 0 & $\begin{array}{l}115 \\
(2,113)\end{array}$ \\
\hline 23513 & 101 & 0 & 0 & $11(0,11)$ & 261 & 1 & 0 & $95(0,95)$ \\
\hline 13589 & 100 & 3 & 0 & $2(1,1)$ & 381 & 14 & 0 & $85(3,82)$ \\
\hline 15267 & 100 & 0 & 0 & $8(0,8)$ & 240 & 1 & 0 & $76(0,76)$ \\
\hline 17479 & 99 & 2 & 0 & $11(1,10)$ & 554 & 5 & 0 & $\begin{array}{l}213 \\
(0,213)\end{array}$ \\
\hline
\end{tabular}


Table 4 (Continued)

\begin{tabular}{|c|c|c|c|c|c|c|c|c|}
\hline & Study eligib & le patients & & & Study inelig & ble patier & & \\
\hline $\begin{array}{l}\text { Clinician } \\
\text { ID }\end{array}$ & $\begin{array}{l}\text { Total no. } \\
\text { of patients }\end{array}$ & $\begin{array}{l}\text { No. of } \\
\text { patients } \\
\text { macro } \\
\text { used }\end{array}$ & $\begin{array}{l}\text { No. of } \\
\text { patients } \\
\text { macro } \\
\text { used more } \\
\text { than once }\end{array}$ & $\begin{array}{l}\text { No. of } \\
\text { patients } \\
\text { statin } \\
\text { prescribed } \\
\text { (macro } \\
\text { used, } \\
\text { macro not } \\
\text { used) }\end{array}$ & $\begin{array}{l}\text { Total no. } \\
\text { of patients }\end{array}$ & $\begin{array}{l}\text { No. of } \\
\text { patients } \\
\text { macro } \\
\text { used }\end{array}$ & $\begin{array}{l}\text { No. of } \\
\text { patients } \\
\text { macro } \\
\text { used more } \\
\text { than once }\end{array}$ & $\begin{array}{l}\text { No. of } \\
\text { patients } \\
\text { statin } \\
\text { prescribed } \\
\text { (macro } \\
\text { used, } \\
\text { macro not } \\
\text { used) }\end{array}$ \\
\hline 5838 & 96 & 11 & 1 & $8(1,7)$ & 439 & 29 & 1 & $\begin{array}{l}147 \\
(2,145)\end{array}$ \\
\hline 14749 & 91 & 3 & 0 & $15(1,14)$ & 336 & 13 & 3 & $\begin{array}{l}117 \\
(2,115)\end{array}$ \\
\hline 15348 & 91 & 5 & 1 & $18(1,17)$ & 358 & 15 & 0 & $\begin{array}{l}126 \\
(2,124)\end{array}$ \\
\hline 9825 & 89 & 11 & 0 & $24(3,21)$ & 373 & 15 & 1 & $\begin{array}{l}163 \\
(1,162)\end{array}$ \\
\hline 7776 & 88 & 0 & 0 & $4(0,4)$ & 222 & 1 & 0 & $58(1,57)$ \\
\hline 25950 & 86 & 4 & 0 & $7(1,6)$ & 371 & 9 & 0 & $95(4,91)$ \\
\hline 30320 & 86 & 0 & 0 & $10(0,10)$ & 540 & 2 & 0 & $\begin{array}{l}106 \\
(0,106)\end{array}$ \\
\hline 31344 & 85 & 7 & 0 & $15(1,14)$ & 324 & 7 & 0 & $87(0,87)$ \\
\hline 18108 & 84 & 5 & 0 & $23(3,20)$ & 562 & 11 & 0 & $\begin{array}{l}204 \\
(1,203)\end{array}$ \\
\hline 26751 & 84 & 1 & 0 & $14(0,14)$ & 400 & 6 & 0 & $\begin{array}{l}153 \\
(2,151) \\
\end{array}$ \\
\hline 14900 & 83 & 4 & 0 & $14(1,13)$ & 423 & 6 & 0 & $\begin{array}{l}132 \\
(3,129)\end{array}$ \\
\hline 31643 & 83 & 0 & 0 & $19(0,19)$ & 284 & 1 & 0 & $\begin{array}{l}103 \\
(0,103)\end{array}$ \\
\hline 30342 & 82 & 0 & 0 & $7(0,7)$ & 560 & 1 & 0 & $97(0,97)$ \\
\hline 30340 & 80 & 3 & 0 & $23(3,20)$ & 831 & 31 & 0 & $\begin{array}{l}207 \\
(10,197)\end{array}$ \\
\hline 28599 & 79 & 4 & 0 & $18(2,16)$ & 307 & 5 & 0 & $\begin{array}{l}103 \\
(3,100) \\
\end{array}$ \\
\hline 17436 & 78 & 0 & 0 & $10(0,10)$ & 420 & 4 & 0 & $\begin{array}{l}153 \\
(0,153)\end{array}$ \\
\hline 17873 & 76 & 9 & 3 & $9(3,6)$ & 517 & 18 & 0 & $\begin{array}{l}119 \\
(2,117)\end{array}$ \\
\hline 19243 & 76 & 6 & 0 & $11(1,10)$ & 275 & 1 & 0 & $\begin{array}{l}101 \\
(1,100)\end{array}$ \\
\hline 30337 & 76 & 5 & 0 & $15(1,14)$ & 563 & 18 & 1 & $\begin{array}{l}107 \\
(2,105)\end{array}$ \\
\hline 31406 & 75 & 3 & 0 & $11(1,10)$ & 411 & 12 & 0 & $\begin{array}{l}128 \\
(1,127)\end{array}$ \\
\hline 19090 & 74 & 5 & 0 & $8(1,7)$ & 315 & 2 & 0 & $63(1,62)$ \\
\hline 6162 & 71 & 2 & 0 & $8(1,7)$ & 474 & 3 & 0 & $\begin{array}{l}180 \\
(1,179)\end{array}$ \\
\hline 14821 & 70 & 1 & 0 & $8(0,8)$ & 186 & 0 & 0 & $74(0,74)$ \\
\hline 27277 & 70 & 11 & 3 & $3(0,3)$ & 322 & 24 & 2 & $98(5,93)$ \\
\hline 30329 & 68 & 0 & 0 & $11(0,11)$ & 687 & 1 & 0 & $\begin{array}{l}178 \\
(0,178)\end{array}$ \\
\hline
\end{tabular}

(Continued) 
Table 4 (Continued)

\begin{tabular}{|c|c|c|c|c|c|c|c|c|}
\hline & Study eligib & e patients & & & Study inelig & ble patien & & \\
\hline $\begin{array}{l}\text { Clinician } \\
\text { ID }\end{array}$ & $\begin{array}{l}\text { Total no. } \\
\text { of patients }\end{array}$ & $\begin{array}{l}\text { No. of } \\
\text { patients } \\
\text { macro } \\
\text { used }\end{array}$ & $\begin{array}{l}\text { No. of } \\
\text { patients } \\
\text { macro } \\
\text { used more } \\
\text { than once }\end{array}$ & $\begin{array}{l}\text { No. of } \\
\text { patients } \\
\text { statin } \\
\text { prescribed } \\
\text { (macro } \\
\text { used, } \\
\text { macro not } \\
\text { used) }\end{array}$ & $\begin{array}{l}\text { Total no. } \\
\text { of patients }\end{array}$ & $\begin{array}{l}\text { No. of } \\
\text { patients } \\
\text { macro } \\
\text { used }\end{array}$ & $\begin{array}{l}\text { No. of } \\
\text { patients } \\
\text { macro } \\
\text { used more } \\
\text { than once }\end{array}$ & $\begin{array}{l}\text { No. of } \\
\text { patients } \\
\text { statin } \\
\text { prescribed } \\
\text { (macro } \\
\text { used, } \\
\text { macro not } \\
\text { used) }\end{array}$ \\
\hline 27338 & 67 & 6 & 1 & $5(0,5)$ & 333 & 18 & 1 & $81(5,76)$ \\
\hline 25053 & 66 & 0 & 0 & $7(0,7)$ & 344 & 2 & 0 & $\begin{array}{l}108 \\
(1,107)\end{array}$ \\
\hline 26849 & 66 & 2 & 0 & $3(0,3)$ & 185 & 4 & 0 & $51(0,51)$ \\
\hline 27632 & 63 & 3 & 1 & $10(0,10)$ & 283 & 4 & 0 & $53(1,52)$ \\
\hline 15000 & 62 & 4 & 1 & $5(2,3)$ & 144 & 2 & 0 & $70(0,70)$ \\
\hline 28934 & 62 & 0 & 0 & $14(0,14)$ & 305 & 11 & 0 & $\begin{array}{l}110 \\
(6,104)\end{array}$ \\
\hline 29225 & 62 & 4 & 0 & $7(1,6)$ & 367 & 7 & 0 & $\begin{array}{l}118 \\
(6,112)\end{array}$ \\
\hline 22153 & 61 & 0 & 0 & $3(0,3)$ & 237 & 10 & 1 & $70(4,66)$ \\
\hline 14891 & 59 & 1 & 0 & $6(0,6)$ & 221 & 0 & 0 & $64(0,64)$ \\
\hline 15240 & 59 & 4 & 1 & $12(1,11)$ & 259 & 9 & 1 & $\begin{array}{l}115 \\
(4,111)\end{array}$ \\
\hline 28297 & 59 & 2 & 0 & $15(1,14)$ & 286 & 6 & 0 & $81(1,80)$ \\
\hline 18276 & 58 & 1 & 0 & $9(0,9)$ & 82 & 2 & 0 & $54(2,52)$ \\
\hline 19733 & 58 & 2 & 0 & $6(1,5)$ & 366 & 5 & 0 & $\begin{array}{l}124 \\
(3,121)\end{array}$ \\
\hline 30311 & 56 & 0 & 0 & $4(0,4)$ & 520 & 2 & 1 & $63(0,63)$ \\
\hline 30332 & 56 & 0 & 0 & $9(0,9)$ & 526 & 1 & 0 & $77(0,77)$ \\
\hline 28044 & 54 & 8 & 0 & $13(1,12)$ & 388 & 14 & 0 & $\begin{array}{l}139 \\
(5,134)\end{array}$ \\
\hline 30327 & 54 & 1 & 0 & $6(0,6)$ & 512 & 28 & 0 & $87(2,85)$ \\
\hline 30341 & 53 & 1 & 0 & $5(0,5)$ & 659 & 35 & 1 & $100(6,94)$ \\
\hline 12997 & 52 & 0 & 0 & $0(0,0)$ & 154 & 2 & 0 & $60(0,60)$ \\
\hline 27079 & 52 & 3 & 1 & $6(2,4)$ & 132 & 3 & 0 & $59(2,57)$ \\
\hline 30322 & 52 & 3 & 2 & $9(0,9)$ & 532 & 34 & 5 & $\begin{array}{l}112 \\
(9,103)\end{array}$ \\
\hline 15384 & 51 & 1 & 0 & $2(0,2)$ & 252 & 7 & 1 & $88(1,87)$ \\
\hline 18673 & 51 & 1 & 0 & $0(0,0)$ & 527 & 0 & 0 & $35(0,35)$ \\
\hline 19506 & 51 & 1 & 0 & $9(0,9)$ & 245 & 0 & 0 & $61(0,61)$ \\
\hline 26777 & 51 & 8 & 0 & $9(1,8)$ & 294 & 27 & 0 & $77(11,66)$ \\
\hline 31365 & 50 & 0 & 0 & $5(0,5)$ & 204 & 1 & 0 & $55(1,54)$ \\
\hline 5996 & 50 & 7 & 0 & $7(2,5)$ & 427 & 30 & 1 & $\begin{array}{l}148 \\
(2,146)\end{array}$ \\
\hline 8183 & 50 & 3 & 0 & $6(0,6)$ & 239 & 5 & 0 & $71(0,71)$ \\
\hline 20912 & 49 & 6 & 1 & $8(0,8)$ & 145 & 3 & 0 & $74(0,74)$ \\
\hline 25156 & 48 & 7 & 3 & $15(3,12)$ & 224 & 23 & 2 & $96(6,90)$ \\
\hline 25372 & 48 & 2 & 0 & $7(1,6)$ & 170 & 1 & 0 & $55(0,55)$ \\
\hline 25720 & 48 & 0 & 0 & $4(0,4)$ & 316 & 7 & 0 & $61(2,59)$ \\
\hline
\end{tabular}


Table 4 (Continued)

\begin{tabular}{|c|c|c|c|c|c|c|c|c|}
\hline \multirow[b]{2}{*}{$\begin{array}{l}\text { Clinician } \\
\text { ID }\end{array}$} & \multicolumn{4}{|c|}{ Study eligible patients } & \multicolumn{4}{|c|}{ Study ineligible patients } \\
\hline & $\begin{array}{l}\text { Total no. } \\
\text { of patients }\end{array}$ & $\begin{array}{l}\text { No. of } \\
\text { patients } \\
\text { macro } \\
\text { used }\end{array}$ & $\begin{array}{l}\text { No. of } \\
\text { patients } \\
\text { macro } \\
\text { used more } \\
\text { than once }\end{array}$ & $\begin{array}{l}\text { No. of } \\
\text { patients } \\
\text { statin } \\
\text { prescribed } \\
\text { (macro } \\
\text { used, } \\
\text { macro not } \\
\text { used) }\end{array}$ & $\begin{array}{l}\text { Total no. } \\
\text { of patients }\end{array}$ & $\begin{array}{l}\text { No. of } \\
\text { patients } \\
\text { macro } \\
\text { used }\end{array}$ & $\begin{array}{l}\text { No. of } \\
\text { patients } \\
\text { macro } \\
\text { used more } \\
\text { than once }\end{array}$ & $\begin{array}{l}\text { No. of } \\
\text { patients } \\
\text { statin } \\
\text { prescribed } \\
\text { (macro } \\
\text { used, } \\
\text { macro not } \\
\text { used) }\end{array}$ \\
\hline 30394 & 48 & 3 & 0 & $15(1,14)$ & 442 & 9 & 0 & $\begin{array}{l}119 \\
(3,116)\end{array}$ \\
\hline 17237 & 47 & 8 & 1 & $12(2,10)$ & 350 & 8 & 0 & $\begin{array}{l}104 \\
(3,101)\end{array}$ \\
\hline 30408 & 47 & 8 & 4 & $16(3,13)$ & 209 & 12 & 1 & $88(3,85)$ \\
\hline 31351 & 47 & 1 & 0 & $2(0,2)$ & 261 & 0 & 0 & $58(0,58)$ \\
\hline 24906 & 46 & 2 & 0 & $14(0,14)$ & 297 & 4 & 1 & $63(0,63)$ \\
\hline 30343 & 46 & 0 & 0 & $6(0,6)$ & 552 & 10 & 1 & $\begin{array}{l}116 \\
(4,112) \\
\end{array}$ \\
\hline 13939 & 45 & 1 & 0 & $4(1,3)$ & 128 & 0 & 0 & $66(0,66)$ \\
\hline 19338 & 45 & 0 & 0 & $9(0,9)$ & 218 & 3 & 0 & $\begin{array}{l}116 \\
(2,114)\end{array}$ \\
\hline 22879 & 45 & 0 & 0 & $1(0,1)$ & 376 & 3 & 0 & $82(0,82)$ \\
\hline 25225 & 43 & 2 & 0 & $3(0,3)$ & 222 & 5 & 0 & $62(0,62)$ \\
\hline 26841 & 43 & 0 & 0 & $4(0,4)$ & 465 & 1 & 0 & $43(0,43)$ \\
\hline 24399 & 42 & 0 & 0 & $6(0,6)$ & 85 & 2 & 0 & $43(1,42)$ \\
\hline 31430 & 42 & 2 & 0 & $9(0,9)$ & 161 & 0 & 0 & $36(0,36)$ \\
\hline 32271 & 42 & 0 & 0 & $5(0,5)$ & 143 & 3 & 0 & $68(2,66)$ \\
\hline 9187 & 42 & 1 & 0 & $3(0,3)$ & 129 & 2 & 0 & $60(0,60)$ \\
\hline 19189 & 41 & 1 & 0 & $8(1,7)$ & 334 & 7 & 0 & $93(2,91)$ \\
\hline 14961 & 40 & 1 & 0 & $4(0,4)$ & 332 & 4 & 0 & $64(0,64)$ \\
\hline 27642 & 39 & 0 & 0 & $9(0,9)$ & 181 & 1 & 0 & $63(1,62)$ \\
\hline 31098 & 39 & 0 & 0 & $2(0,2)$ & 414 & 2 & 0 & $37(1,36)$ \\
\hline 30335 & 37 & 0 & 0 & $9(0,9)$ & 301 & 1 & 0 & $65(1,64)$ \\
\hline 23235 & 36 & 1 & 0 & $5(0,5)$ & 286 & 1 & 0 & $51(0,51)$ \\
\hline 15866 & 35 & 3 & 0 & $10(2,8)$ & 244 & 19 & 1 & $\begin{array}{l}115 \\
(6,109)\end{array}$ \\
\hline 18117 & 35 & 2 & 0 & $9(0,9)$ & 192 & 11 & 3 & $41(0,41)$ \\
\hline 31644 & 35 & 2 & 0 & $6(1,5)$ & 244 & 17 & 0 & $54(4,50)$ \\
\hline 11213 & 34 & 1 & 0 & $8(0,8)$ & 106 & 1 & 0 & $48(0,48)$ \\
\hline 22866 & 34 & 2 & 0 & $5(1,4)$ & 250 & 3 & 0 & $62(0,62)$ \\
\hline 27268 & 34 & 1 & 1 & $8(1,7)$ & 211 & 7 & 0 & $55(2,53)$ \\
\hline 23603 & 33 & 1 & 0 & $0(0,0)$ & 163 & 0 & 0 & $20(0,20)$ \\
\hline 31840 & 33 & 2 & 0 & $11(1,10)$ & 168 & 11 & 0 & $32(2,30)$ \\
\hline 13272 & 32 & 0 & 0 & $7(0,7)$ & 482 & 2 & 0 & $69(0,69)$ \\
\hline 29296 & 32 & 2 & 0 & $11(2,9)$ & 123 & 4 & 0 & $44(1,43)$ \\
\hline 8494 & 32 & 1 & 0 & $6(0,6)$ & 75 & 1 & 0 & $40(1,39)$ \\
\hline 24383 & 30 & 2 & 1 & $4(0,4)$ & 141 & 5 & 0 & $46(0,46)$ \\
\hline 6308 & 30 & 4 & 0 & $5(0,5)$ & 72 & 4 & 0 & $54(2,52)$ \\
\hline
\end{tabular}


Table 4 (Continued)

\begin{tabular}{|c|c|c|c|c|c|c|c|c|}
\hline & Study eligit & le patients & & & Study inelig & ble patien & & \\
\hline $\begin{array}{l}\text { Clinician } \\
\text { ID }\end{array}$ & $\begin{array}{l}\text { Total no. } \\
\text { of patients }\end{array}$ & $\begin{array}{l}\text { No. of } \\
\text { patients } \\
\text { macro } \\
\text { used }\end{array}$ & $\begin{array}{l}\text { No. of } \\
\text { patients } \\
\text { macro } \\
\text { used more } \\
\text { than once }\end{array}$ & 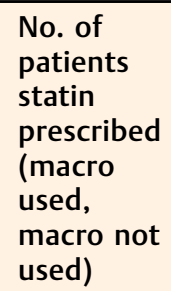 & $\begin{array}{l}\text { Total no. } \\
\text { of patients }\end{array}$ & $\begin{array}{l}\text { No. of } \\
\text { patients } \\
\text { macro } \\
\text { used }\end{array}$ & $\begin{array}{l}\text { No. of } \\
\text { patients } \\
\text { macro } \\
\text { used more } \\
\text { than once }\end{array}$ & $\begin{array}{l}\text { No. of } \\
\text { patients } \\
\text { statin } \\
\text { prescribed } \\
\text { (macro } \\
\text { used, } \\
\text { macro not } \\
\text { used) }\end{array}$ \\
\hline Missing & 29 & 1 & 0 & $3(0,3)$ & 160 & 4 & 0 & $46(0,46)$ \\
\hline 12428 & 29 & 2 & 0 & $5(1,4)$ & 282 & 24 & 3 & $68(7,61)$ \\
\hline 19384 & 29 & 4 & 0 & $6(0,6)$ & 96 & 3 & 0 & $57(0,57)$ \\
\hline 10399 & 28 & 0 & 0 & $5(0,5)$ & 243 & 1 & 0 & $42(0,42)$ \\
\hline 13600 & 27 & 1 & 0 & $1(0,1)$ & 79 & 1 & 0 & $39(1,38)$ \\
\hline 15251 & 27 & 2 & 1 & $7(1,6)$ & 161 & 1 & 0 & $55(0,55)$ \\
\hline 19691 & 27 & 0 & 0 & $3(0,3)$ & 69 & 1 & 0 & $25(1,24)$ \\
\hline 30324 & 27 & 0 & 0 & $2(0,2)$ & 443 & 4 & 0 & $75(0,75)$ \\
\hline 17448 & 25 & 0 & 0 & $2(0,2)$ & 176 & 5 & 0 & $39(0,39)$ \\
\hline 30336 & 25 & 0 & 0 & $4(0,4)$ & 419 & 20 & 1 & $35(3,32)$ \\
\hline 27839 & 24 & 3 & 0 & $3(0,3)$ & 64 & 0 & 0 & $25(0,25)$ \\
\hline 25241 & 23 & 0 & 0 & $6(0,6)$ & 132 & 5 & 1 & $63(1,62)$ \\
\hline 28109 & 22 & 1 & 0 & $9(0,9)$ & 182 & 15 & 1 & $55(4,51)$ \\
\hline 31420 & 22 & 1 & 0 & $2(1,1)$ & 331 & 10 & 1 & $35(0,35)$ \\
\hline 9849 & 22 & 1 & 0 & $2(0,2)$ & 45 & 3 & 0 & $15(1,14)$ \\
\hline 26833 & 21 & 0 & 0 & $2(0,2)$ & 147 & 2 & 0 & $38(0,38)$ \\
\hline 32040 & 21 & 0 & 0 & $3(0,3)$ & 361 & 1 & 0 & $29(0,29)$ \\
\hline 15286 & 20 & 1 & 0 & $2(0,2)$ & 110 & 4 & 1 & $24(1,23)$ \\
\hline 19168 & 20 & 0 & 0 & $1(0,1)$ & 66 & 1 & 0 & $24(0,24)$ \\
\hline 21774 & 20 & 1 & 1 & $3(0,3)$ & 128 & 2 & 0 & $27(0,27)$ \\
\hline 27355 & 20 & 0 & 0 & $2(0,2)$ & 367 & 1 & 0 & $50(0,50)$ \\
\hline 28133 & 19 & 3 & 2 & $7(1,6)$ & 90 & 7 & 0 & $35(1,34)$ \\
\hline 28428 & 17 & 0 & 0 & $0(0,0)$ & 66 & 1 & 0 & $6(0,6)$ \\
\hline 26839 & 16 & 0 & 0 & $1(0,1)$ & 119 & 2 & 0 & $25(1,24)$ \\
\hline 26042 & 15 & 2 & 0 & $2(1,1)$ & 65 & 1 & 0 & $1(0,1)$ \\
\hline 6806 & 15 & 1 & 0 & $1(0,1)$ & 24 & 0 & 0 & $16(0,16)$ \\
\hline 26041 & 14 & 1 & 0 & $6(1,5)$ & 79 & 4 & 0 & $23(1,22)$ \\
\hline 9719 & 14 & 1 & 0 & $4(1,3)$ & 33 & 3 & 0 & $11(1,10)$ \\
\hline 22922 & 13 & 0 & 0 & $2(0,2)$ & 69 & 1 & 0 & $21(1,20)$ \\
\hline 25948 & 13 & 0 & 0 & $1(0,1)$ & 56 & 5 & 0 & $25(1,24)$ \\
\hline 28117 & 13 & 0 & 0 & $1(0,1)$ & 41 & 4 & 0 & $6(2,4)$ \\
\hline 90000005 & 13 & 0 & 0 & $1(0,1)$ & 109 & 1 & 0 & $26(0,26)$ \\
\hline 16367 & 12 & 4 & 1 & $4(0,4)$ & 32 & 4 & 0 & $9(0,9)$ \\
\hline 28040 & 12 & 1 & 0 & $1(0,1)$ & 75 & 6 & 0 & $20(2,18)$ \\
\hline 31014 & 12 & 1 & 0 & $2(0,2)$ & 104 & 5 & 0 & $16(1,15)$ \\
\hline 28039 & 11 & 0 & 0 & $1(0,1)$ & 89 & 5 & 0 & $18(0,18)$ \\
\hline 21032 & 10 & 1 & 0 & $4(0,4)$ & 47 & 0 & 0 & $18(0,18)$ \\
\hline 22142 & 10 & 2 & 1 & $1(0,1)$ & 22 & 0 & 0 & $12(0,12)$ \\
\hline
\end{tabular}


Table 4 (Continued)

\begin{tabular}{|c|c|c|c|c|c|c|c|c|}
\hline \multirow[b]{2}{*}{$\begin{array}{l}\text { Clinician } \\
\text { ID }\end{array}$} & \multicolumn{4}{|c|}{ Study eligible patients } & \multicolumn{4}{|c|}{ Study ineligible patients } \\
\hline & $\begin{array}{l}\text { Total no. } \\
\text { of patients }\end{array}$ & $\begin{array}{l}\text { No. of } \\
\text { patients } \\
\text { macro } \\
\text { used }\end{array}$ & $\begin{array}{l}\text { No. of } \\
\text { patients } \\
\text { macro } \\
\text { used more } \\
\text { than once }\end{array}$ & $\begin{array}{l}\text { No. of } \\
\text { patients } \\
\text { statin } \\
\text { prescribed } \\
\text { (macro } \\
\text { used, } \\
\text { macro not } \\
\text { used) }\end{array}$ & $\begin{array}{l}\text { Total no. } \\
\text { of patients }\end{array}$ & $\begin{array}{l}\text { No. of } \\
\text { patients } \\
\text { macro } \\
\text { used }\end{array}$ & $\begin{array}{l}\text { No. of } \\
\text { patients } \\
\text { macro } \\
\text { used more } \\
\text { than once }\end{array}$ & $\begin{array}{l}\text { No. of } \\
\text { patients } \\
\text { statin } \\
\text { prescribed } \\
\text { (macro } \\
\text { used, } \\
\text { macro not } \\
\text { used) }\end{array}$ \\
\hline 26838 & 10 & 3 & 0 & $1(1,0)$ & 49 & 3 & 0 & $19(1,18)$ \\
\hline 12413 & 9 & 2 & 0 & $0(0,0)$ & 32 & 1 & 0 & $16(1,15)$ \\
\hline 16570 & 9 & 0 & 0 & $0(0,0)$ & 41 & 1 & 0 & $8(0,8)$ \\
\hline 27912 & 9 & 2 & 0 & $1(1,0)$ & 26 & 3 & 1 & $9(0,9)$ \\
\hline 28857 & 9 & 0 & 0 & $1(0,1)$ & 33 & 1 & 0 & $5(1,4)$ \\
\hline 29484 & 9 & 0 & 0 & $3(0,3)$ & 64 & 2 & 0 & $17(1,16)$ \\
\hline 18575 & 8 & 0 & 0 & $1(0,1)$ & 26 & 1 & 0 & $9(0,9)$ \\
\hline 18661 & 8 & 1 & 0 & $1(0,1)$ & 27 & 3 & 0 & $14(1,13)$ \\
\hline 31977 & 8 & 0 & 0 & $3(0,3)$ & 19 & 1 & 0 & $10(1,9)$ \\
\hline 5944 & 8 & 1 & 0 & $0(0,0)$ & 9 & 0 & 0 & $6(0,6)$ \\
\hline 13089 & 7 & 1 & 1 & $1(0,1)$ & 35 & 3 & 0 & $11(1,10)$ \\
\hline 21782 & 7 & 1 & 0 & $0(0,0)$ & 10 & 1 & 0 & $5(1,4)$ \\
\hline 32336 & 7 & 1 & 0 & $2(0,2)$ & 12 & 0 & 0 & $3(0,3)$ \\
\hline 31975 & 6 & 0 & 0 & $0(0,0)$ & 202 & 1 & 0 & $11(0,11)$ \\
\hline 32626 & 6 & 0 & 0 & $3(0,3)$ & 34 & 1 & 0 & $9(1,8)$ \\
\hline 27207 & 5 & 1 & 0 & $2(0,2)$ & 28 & 2 & 0 & $2(0,2)$ \\
\hline 28878 & 5 & 0 & 0 & $0(0,0)$ & 119 & 13 & 0 & $32(3,29)$ \\
\hline 31849 & 5 & 0 & 0 & $3(0,3)$ & 93 & 2 & 0 & $23(1,22)$ \\
\hline 28883 & 4 & 0 & 0 & $1(0,1)$ & 21 & 1 & 0 & $6(0,6)$ \\
\hline 30488 & 4 & 0 & 0 & $0(0,0)$ & 50 & 1 & 0 & $10(0,10)$ \\
\hline 27471 & 3 & 1 & 0 & $0(0,0)$ & 9 & 0 & 0 & $1(0,1)$ \\
\hline 10682 & 2 & 0 & 0 & $1(0,1)$ & 2 & 1 & 0 & $2(1,1)$ \\
\hline 13088 & 2 & 0 & 0 & $0(0,0)$ & 19 & 2 & 0 & $6(0,6)$ \\
\hline 16535 & 2 & 0 & 0 & $1(0,1)$ & 9 & 1 & 1 & $5(1,4)$ \\
\hline 19413 & 2 & 0 & 0 & $1(0,1)$ & 47 & 1 & 0 & $8(0,8)$ \\
\hline 29736 & 2 & 0 & 0 & $1(0,1)$ & 7 & 1 & 0 & $6(0,6)$ \\
\hline 30640 & 2 & 1 & 0 & $0(0,0)$ & 9 & 2 & 0 & $6(2,4)$ \\
\hline 30647 & 2 & 0 & 0 & $1(0,1)$ & 10 & 2 & 0 & $1(0,1)$ \\
\hline 30656 & 2 & 1 & 0 & $1(1,0)$ & 5 & 0 & 0 & $3(0,3)$ \\
\hline 31340 & 2 & 0 & 0 & $0(0,0)$ & 54 & 2 & 0 & $9(1,8)$ \\
\hline 31574 & 2 & 1 & 1 & $2(1,1)$ & 6 & 0 & 0 & $1(0,1)$ \\
\hline 31583 & 2 & 0 & 0 & $0(0,0)$ & 6 & 1 & 0 & $4(1,3)$ \\
\hline 31598 & 2 & 1 & 0 & $0(0,0)$ & 8 & 1 & 0 & $5(1,4)$ \\
\hline 32041 & 2 & 0 & 0 & $0(0,0)$ & 28 & 1 & 1 & $0(0,0)$ \\
\hline 32285 & 2 & 0 & 0 & $1(0,1)$ & 33 & 1 & 0 & $10(0,10)$ \\
\hline 32340 & 2 & 0 & 0 & $0(0,0)$ & 49 & 4 & 0 & $9(0,9)$ \\
\hline 32462 & 2 & 0 & 0 & $0(0,0)$ & 5 & 1 & 0 & $3(0,3)$ \\
\hline 3860 & 2 & 0 & 0 & $0(0,0)$ & 185 & 1 & 0 & $0(0,0)$ \\
\hline
\end{tabular}

(Continued) 
Table 4 (Continued)

\begin{tabular}{|c|c|c|c|c|c|c|c|c|}
\hline & Study eligib & e patients & & & Study inelig & ble patien & & \\
\hline $\begin{array}{l}\text { Clinician } \\
\text { ID }\end{array}$ & $\begin{array}{l}\text { Total no. } \\
\text { of patients }\end{array}$ & $\begin{array}{l}\text { No. of } \\
\text { patients } \\
\text { macro } \\
\text { used }\end{array}$ & $\begin{array}{l}\text { No. of } \\
\text { patients } \\
\text { macro } \\
\text { used more } \\
\text { than once }\end{array}$ & 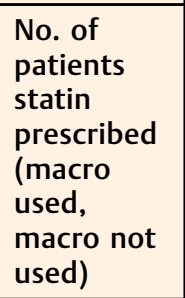 & $\begin{array}{l}\text { Total no. } \\
\text { of patients }\end{array}$ & $\begin{array}{l}\text { No. of } \\
\text { patients } \\
\text { macro } \\
\text { used }\end{array}$ & $\begin{array}{l}\text { No. of } \\
\text { patients } \\
\text { macro } \\
\text { used more } \\
\text { than once }\end{array}$ & $\begin{array}{l}\text { No. of } \\
\text { patients } \\
\text { statin } \\
\text { prescribed } \\
\text { (macro } \\
\text { used, } \\
\text { macro not } \\
\text { used) }\end{array}$ \\
\hline 28858 & 1 & 0 & 0 & $0(0,0)$ & 9 & 1 & 0 & $4(1,3)$ \\
\hline 29806 & 1 & 0 & 0 & $0(0,0)$ & 11 & 2 & 0 & $6(1,5)$ \\
\hline 30664 & 1 & 0 & 0 & $1(0,1)$ & 12 & 1 & 0 & $7(1,6)$ \\
\hline 30666 & 1 & 0 & 0 & $1(0,1)$ & 11 & 2 & 0 & $5(0,5)$ \\
\hline 30694 & 1 & 1 & 0 & $1(1,0)$ & 1 & 1 & 0 & $0(0,0)$ \\
\hline 31560 & 1 & 1 & 0 & $1(1,0)$ & 2 & 0 & 0 & $0(0,0)$ \\
\hline 31586 & 1 & 0 & 0 & $0(0,0)$ & 7 & 1 & 0 & $5(1,4)$ \\
\hline 31596 & 1 & 0 & 0 & $0(0,0)$ & 10 & 1 & 0 & $2(0,2)$ \\
\hline 32504 & 1 & 0 & 0 & $0(0,0)$ & 1 & 1 & 0 & $1(1,0)$ \\
\hline 7097 & 1 & 1 & 0 & $1(1,0)$ & 4 & 0 & 0 & $3(0,3)$ \\
\hline 10003848 & 0 & 0 & 0 & $0(0,0)$ & 3 & 1 & 0 & $1(1,0)$ \\
\hline 19799 & 0 & 0 & 0 & $0(0,0)$ & 1 & 1 & 0 & $1(1,0)$ \\
\hline 21750 & 0 & 0 & 0 & $0(0,0)$ & 17 & 1 & 0 & $9(0,9)$ \\
\hline 28938 & 0 & 0 & 0 & $0(0,0)$ & 2 & 1 & 0 & $0(0,0)$ \\
\hline 29800 & 0 & 0 & 0 & $0(0,0)$ & 2 & 1 & 0 & $0(0,0)$ \\
\hline 30326 & 0 & 0 & 0 & $0(0,0)$ & 41 & 1 & 0 & $2(0,2)$ \\
\hline 30584 & 0 & 0 & 0 & $0(0,0)$ & 3 & 1 & 0 & $3(1,2)$ \\
\hline 30652 & 0 & 0 & 0 & $0(0,0)$ & 10 & 2 & 0 & $4(2,2)$ \\
\hline 30662 & 0 & 0 & 0 & $0(0,0)$ & 5 & 1 & 0 & $2(1,1)$ \\
\hline 30672 & 0 & 0 & 0 & $0(0,0)$ & 8 & 1 & 0 & $5(1,4)$ \\
\hline Totals & 9,515 & 389 & 43 & $\begin{array}{l}1218(108, \\
1,110)\end{array}$ & 47,345 & 1,112 & 46 & $\begin{array}{l}13,269 \\
(272, \\
12,997)\end{array}$ \\
\hline
\end{tabular}

Abbreviation: ID, identification.

patients for whom the statin macro was used (odds ratio 2.77, 95\% CI, 2.16-3.54, $p<0.001$ ) while controlling for covariates and clinician in a mixed effect model. Also from -Table 4, for the study ineligible patients, those same physicians used the statin macro 1,112 times to generate 272 statin prescriptions (24\%).

Missing data imputation analysis yielded similar results with our primary analysis. Although more patients met the inclusion/exclusion criteria (11,877 primary analysis vs. 20,240 imputed analysis), the relative risk of statin therapy prescription for macro usage and statin macro odds ratio in the mixed effect logistic regression were similar (see -Supplementary Material, available in the online version).

\section{Discussion}

This study described the implementation of a CDS tool, the statin macro, for the 2013 ACC/AHA statin guideline. For patients recommended but not prescribed statin therapy before the study period, statin macro usage was significantly associated with increased statin prescription during the study period, although the study did not determine if the statin dosages were guideline compliant. This study is the first to show that a 2013 ACC/AHA statin guideline CDS tool was associated with improved statin guideline-related prescription rates. The study cannot establish a cause and effect relationship, in that clinicians might have used the statin macro more frequently after already having decided to prescribe a statin.

Baseline characteristics including DM and LDL were significantly different in patients for whom the statin macro was and was not used. The large sample size lead to statistical significance, but the clinical significance of a $4 \mathrm{mg} / \mathrm{dL}$ difference in LDL levels (123 vs. $127 \mathrm{mg} / \mathrm{dL}$ ) is somewhat trivial. Clinicians may have used the statin macro less often for DM patients as they knew such patients should receive statin therapy. 
Table 5 For clinicians who never used the statin macro and prescribed statin therapy at least once, clinician statin macro usage and prescription stratified by patient study eligibility

\begin{tabular}{|c|c|c|c|c|}
\hline \multirow[t]{2}{*}{ Clinician ID } & \multicolumn{2}{|l|}{ Study eligible patients } & \multicolumn{2}{|c|}{ Study ineligible } \\
\hline & Total no. of patients & $\begin{array}{l}\text { No. of patients } \\
\text { statin prescribed }\end{array}$ & $\begin{array}{l}\text { Total no. of } \\
\text { patients }\end{array}$ & $\begin{array}{l}\text { No. of patients } \\
\text { statin prescribed }\end{array}$ \\
\hline 14823 & 197 & 14 & 550 & 207 \\
\hline 19002 & 152 & 12 & 356 & 136 \\
\hline 15255 & 117 & 9 & 382 & 127 \\
\hline 26250 & 93 & 5 & 451 & 168 \\
\hline 9561 & 91 & 4 & 301 & 101 \\
\hline 30321 & 70 & 8 & 543 & 102 \\
\hline 9102 & 70 & 9 & 492 & 118 \\
\hline 31052 & 69 & 12 & 315 & 86 \\
\hline 14892 & 67 & 3 & 207 & 63 \\
\hline 25268 & 61 & 7 & 209 & 55 \\
\hline 19052 & 59 & 3 & 289 & 53 \\
\hline 7389 & 56 & 12 & 575 & 151 \\
\hline 30319 & 54 & 13 & 658 & 190 \\
\hline 3502 & 50 & 3 & 123 & 32 \\
\hline 6020 & 46 & 6 & 144 & 77 \\
\hline 25083 & 43 & 4 & 313 & 40 \\
\hline 31022 & 38 & 5 & 416 & 40 \\
\hline 32078 & 38 & 7 & 116 & 53 \\
\hline 23205 & 32 & 1 & 59 & 39 \\
\hline 13661 & 30 & 3 & 157 & 42 \\
\hline 29620 & 28 & 2 & 47 & 26 \\
\hline 31184 & 26 & 4 & 220 & 39 \\
\hline 24257 & 25 & 3 & 146 & 79 \\
\hline 31148 & 25 & 2 & 98 & 34 \\
\hline 26023 & 24 & 2 & 198 & 33 \\
\hline 28660 & 24 & 3 & 193 & 49 \\
\hline 31011 & 23 & 2 & 341 & 23 \\
\hline 4294 & 23 & 3 & 63 & 34 \\
\hline 28747 & 22 & 3 & 122 & 28 \\
\hline 30291 & 22 & 3 & 122 & 23 \\
\hline 14704 & 19 & 2 & 36 & 13 \\
\hline 26731 & 17 & 2 & 48 & 31 \\
\hline 30318 & 17 & 2 & 146 & 30 \\
\hline 21251 & 16 & 1 & 357 & 24 \\
\hline 24646 & 16 & 2 & 32 & 16 \\
\hline 28050 & 16 & 1 & 104 & 22 \\
\hline 31754 & 16 & 5 & 82 & 31 \\
\hline 30292 & 14 & 4 & 298 & 41 \\
\hline 32385 & 14 & 5 & 58 & 19 \\
\hline 28048 & 13 & 2 & 135 & 32 \\
\hline 13583 & 12 & 1 & 61 & 32 \\
\hline
\end{tabular}


Table 5 (Continued)

\begin{tabular}{|c|c|c|c|c|}
\hline \multirow[t]{2}{*}{ Clinician ID } & \multicolumn{2}{|l|}{ Study eligible patients } & \multicolumn{2}{|c|}{ Study ineligible } \\
\hline & Total no. of patients & $\begin{array}{l}\text { No. of patients } \\
\text { statin prescribed }\end{array}$ & $\begin{array}{l}\text { Total no. of } \\
\text { patients }\end{array}$ & $\begin{array}{l}\text { No. of patients } \\
\text { statin prescribed }\end{array}$ \\
\hline 18591 & 12 & 0 & 27 & 12 \\
\hline 27779 & 11 & 1 & 47 & 14 \\
\hline 25376 & 10 & 4 & 82 & 8 \\
\hline 26830 & 10 & 1 & 12 & 7 \\
\hline 30382 & 10 & 1 & 37 & 15 \\
\hline 32005 & 10 & 0 & 125 & 7 \\
\hline 14767 & 9 & 2 & 31 & 18 \\
\hline 17325 & 9 & 1 & 53 & 32 \\
\hline 29279 & 9 & 0 & 19 & 12 \\
\hline 30288 & 9 & 2 & 229 & 14 \\
\hline 6494 & 9 & 0 & 29 & 14 \\
\hline 27456 & 8 & 0 & 13 & 12 \\
\hline 8251 & 8 & 0 & 10 & 5 \\
\hline 10381 & 7 & 0 & 28 & 13 \\
\hline 16407 & 7 & 2 & 19 & 11 \\
\hline 21366 & 7 & 1 & 17 & 8 \\
\hline 24204 & 7 & 1 & 23 & 13 \\
\hline 30379 & 7 & 0 & 39 & 23 \\
\hline 31794 & 7 & 0 & 41 & 4 \\
\hline 32278 & 7 & 0 & 25 & 9 \\
\hline 16391 & 6 & 0 & 5 & 4 \\
\hline 17319 & 6 & 2 & 95 & 30 \\
\hline 17792 & 6 & 1 & 24 & 7 \\
\hline 18273 & 6 & 0 & 14 & 9 \\
\hline 2619 & 5 & 0 & 15 & 3 \\
\hline 28089 & 5 & 2 & 30 & 10 \\
\hline 28611 & 5 & 1 & 4 & 3 \\
\hline 30317 & 5 & 0 & 32 & 11 \\
\hline 30677 & 5 & 2 & 5 & 1 \\
\hline 32357 & 5 & 0 & 13 & 6 \\
\hline 32961 & 5 & 0 & 11 & 6 \\
\hline 14275 & 4 & 0 & 26 & 3 \\
\hline 16953 & 4 & 1 & 2 & 0 \\
\hline 24969 & 4 & 0 & 5 & 2 \\
\hline 26389 & 4 & 0 & 37 & 16 \\
\hline 29741 & 4 & 0 & 20 & 5 \\
\hline 29816 & 4 & 1 & 25 & 14 \\
\hline 30651 & 4 & 1 & 10 & 4 \\
\hline 31591 & 4 & 0 & 10 & 2 \\
\hline 13656 & 3 & 2 & 40 & 24 \\
\hline 14756 & 3 & 1 & 4 & 3 \\
\hline 18769 & 3 & 0 & 19 & 14 \\
\hline
\end{tabular}


Table 5 (Continued)

\begin{tabular}{|c|c|c|c|c|}
\hline \multirow[t]{2}{*}{ Clinician ID } & \multicolumn{2}{|l|}{ Study eligible patients } & \multicolumn{2}{|c|}{ Study ineligible } \\
\hline & Total no. of patients & $\begin{array}{l}\text { No. of patients } \\
\text { statin prescribed }\end{array}$ & $\begin{array}{l}\text { Total no. of } \\
\text { patients }\end{array}$ & $\begin{array}{l}\text { No. of patients } \\
\text { statin prescribed }\end{array}$ \\
\hline 27330 & 3 & 0 & 6 & 3 \\
\hline 27820 & 3 & 0 & 37 & 3 \\
\hline 29649 & 3 & 0 & 8 & 4 \\
\hline 29809 & 3 & 0 & 5 & 2 \\
\hline 30210 & 3 & 1 & 49 & 1 \\
\hline 30644 & 3 & 0 & 10 & 3 \\
\hline 30650 & 3 & 1 & 5 & 1 \\
\hline 31398 & 3 & 1 & 8 & 2 \\
\hline 31566 & 3 & 0 & 4 & 3 \\
\hline 31577 & 3 & 0 & 6 & 6 \\
\hline 10887 & 2 & 1 & 7 & 5 \\
\hline 13093 & 2 & 1 & 4 & 2 \\
\hline 14765 & 2 & 0 & 1 & 1 \\
\hline 15448 & 2 & 1 & 8 & 4 \\
\hline 17383 & 2 & 0 & 7 & 1 \\
\hline 17651 & 2 & 0 & 5 & 1 \\
\hline 20928 & 2 & 1 & 9 & 5 \\
\hline 21472 & 2 & 0 & 4 & 1 \\
\hline 21971 & 2 & 0 & 16 & 1 \\
\hline 26465 & 2 & 1 & 4 & 0 \\
\hline 28148 & 2 & 0 & 8 & 5 \\
\hline 28617 & 2 & 0 & 7 & 2 \\
\hline 29794 & 2 & 1 & 13 & 5 \\
\hline 29818 & 2 & 0 & 8 & 4 \\
\hline 30626 & 2 & 0 & 7 & 3 \\
\hline 30636 & 2 & 0 & 7 & 1 \\
\hline 30641 & 2 & 0 & 9 & 3 \\
\hline 30665 & 2 & 0 & 5 & 1 \\
\hline 31573 & 2 & 0 & 6 & 2 \\
\hline 32474 & 2 & 1 & 4 & 3 \\
\hline 32966 & 2 & 0 & 29 & 6 \\
\hline 33145 & 2 & 0 & 16 & 7 \\
\hline 7020 & 2 & 0 & 61 & 10 \\
\hline 7773 & 2 & 0 & 4 & 2 \\
\hline 9183 & 2 & 0 & 15 & 1 \\
\hline 10001334 & 1 & 1 & 0 & 0 \\
\hline 10115 & 1 & 1 & 0 & 0 \\
\hline 11416 & 1 & 0 & 16 & 1 \\
\hline 15404 & 1 & 0 & 3 & 1 \\
\hline 18103 & 1 & 0 & 7 & 4 \\
\hline 20170 & 1 & 0 & 1 & 1 \\
\hline 20373 & 1 & 0 & 3 & 1 \\
\hline
\end{tabular}

(Continued) 
Table 5 (Continued)

\begin{tabular}{|c|c|c|c|c|}
\hline \multirow[t]{2}{*}{ Clinician ID } & \multicolumn{2}{|l|}{ Study eligible patients } & \multicolumn{2}{|c|}{ Study ineligible } \\
\hline & Total no. of patients & $\begin{array}{l}\text { No. of patients } \\
\text { statin prescribed }\end{array}$ & $\begin{array}{l}\text { Total no. of } \\
\text { patients }\end{array}$ & $\begin{array}{l}\text { No. of patients } \\
\text { statin prescribed }\end{array}$ \\
\hline 21033 & 1 & 0 & 3 & 3 \\
\hline 21134 & 1 & 0 & 8 & 1 \\
\hline 21746 & 1 & 0 & 10 & 1 \\
\hline 22769 & 1 & 0 & 10 & 4 \\
\hline 22802 & 1 & 0 & 2 & 1 \\
\hline 23153 & 1 & 0 & 6 & 3 \\
\hline 23424 & 1 & 1 & 2 & 1 \\
\hline 23436 & 1 & 0 & 16 & 2 \\
\hline 25144 & 1 & 0 & 3 & 2 \\
\hline 25963 & 1 & 0 & 10 & 2 \\
\hline 28045 & 1 & 0 & 3 & 1 \\
\hline 28677 & 1 & 0 & 11 & 5 \\
\hline 28914 & 1 & 0 & 4 & 3 \\
\hline 29738 & 1 & 0 & 2 & 1 \\
\hline 29742 & 1 & 0 & 2 & 2 \\
\hline 29743 & 1 & 0 & 2 & 2 \\
\hline 29786 & 1 & 0 & 7 & 1 \\
\hline 29788 & 1 & 0 & 2 & 2 \\
\hline 29795 & 1 & 0 & 1 & 1 \\
\hline 29829 & 1 & 0 & 3 & 3 \\
\hline 30333 & 1 & 1 & 119 & 11 \\
\hline 30581 & 1 & 0 & 2 & 1 \\
\hline 30587 & 1 & 0 & 2 & 1 \\
\hline 30646 & 1 & 0 & 10 & 3 \\
\hline 30653 & 1 & 1 & 9 & 3 \\
\hline 30659 & 1 & 0 & 6 & 6 \\
\hline 30660 & 1 & 0 & 6 & 3 \\
\hline 30661 & 1 & 0 & 13 & 3 \\
\hline 30674 & 1 & 0 & 14 & 8 \\
\hline 30678 & 1 & 0 & 10 & 7 \\
\hline 30679 & 1 & 0 & 8 & 4 \\
\hline 31173 & 1 & 0 & 2 & 1 \\
\hline 31556 & 1 & 0 & 3 & 2 \\
\hline 31559 & 1 & 1 & 6 & 3 \\
\hline 31568 & 1 & 1 & 3 & 1 \\
\hline 31570 & 1 & 1 & 4 & 3 \\
\hline 31571 & 1 & 0 & 6 & 2 \\
\hline 31578 & 1 & 0 & 4 & 1 \\
\hline 32004 & 1 & 0 & 24 & 5 \\
\hline 32290 & 1 & 0 & 10 & 2 \\
\hline 32467 & 1 & 0 & 2 & 1 \\
\hline 32472 & 1 & 0 & 4 & 2 \\
\hline
\end{tabular}


Table 5 (Continued)

\begin{tabular}{|c|c|c|c|c|}
\hline \multirow[t]{2}{*}{ Clinician ID } & \multicolumn{2}{|l|}{ Study eligible patients } & \multicolumn{2}{|c|}{ Study ineligible } \\
\hline & Total no. of patients & $\begin{array}{l}\text { No. of patients } \\
\text { statin prescribed }\end{array}$ & $\begin{array}{l}\text { Total no. of } \\
\text { patients }\end{array}$ & $\begin{array}{l}\text { No. of patients } \\
\text { statin prescribed }\end{array}$ \\
\hline 32482 & 1 & 1 & 2 & 2 \\
\hline 32487 & 1 & 0 & 8 & 4 \\
\hline 32497 & 1 & 0 & 2 & 2 \\
\hline 32674 & 1 & 0 & 10 & 3 \\
\hline 33009 & 1 & 0 & 3 & 1 \\
\hline 4392 & 1 & 0 & 3 & 3 \\
\hline 5606 & 1 & 0 & 2 & 1 \\
\hline 8995 & 1 & 0 & 122 & 7 \\
\hline 90000014 & 1 & 0 & 5 & 3 \\
\hline 10000141 & 0 & 0 & 1 & 1 \\
\hline 10000390 & 0 & 0 & 1 & 1 \\
\hline 10000739 & 0 & 0 & 1 & 1 \\
\hline 10001141 & 0 & 0 & 1 & 1 \\
\hline 10004278 & 0 & 0 & 1 & 1 \\
\hline 10004338 & 0 & 0 & 1 & 1 \\
\hline 10004367 & 0 & 0 & 2 & 1 \\
\hline 10004906 & 0 & 0 & 1 & 1 \\
\hline 10007567 & 0 & 0 & 1 & 1 \\
\hline 10009733 & 0 & 0 & 1 & 1 \\
\hline 10011977 & 0 & 0 & 1 & 1 \\
\hline 10022155 & 0 & 0 & 1 & 1 \\
\hline 10022969 & 0 & 0 & 1 & 1 \\
\hline 10030040 & 0 & 0 & 2 & 1 \\
\hline 10033676 & 0 & 0 & 1 & 1 \\
\hline 10034012 & 0 & 0 & 1 & 1 \\
\hline 10041394 & 0 & 0 & 1 & 1 \\
\hline 10044676 & 0 & 0 & 1 & 1 \\
\hline 10047841 & 0 & 0 & 1 & 1 \\
\hline 11000316 & 0 & 0 & 1 & 1 \\
\hline 11000496 & 0 & 0 & 1 & 1 \\
\hline 11000872 & 0 & 0 & 1 & 1 \\
\hline 11001242 & 0 & 0 & 1 & 1 \\
\hline 11006889 & 0 & 0 & 3 & 1 \\
\hline 11011013 & 0 & 0 & 1 & 1 \\
\hline 11012330 & 0 & 0 & 1 & 1 \\
\hline 11013507 & 0 & 0 & 1 & 1 \\
\hline 11016417 & 0 & 0 & 1 & 1 \\
\hline 11017524 & 0 & 0 & 1 & 1 \\
\hline 11029330 & 0 & 0 & 1 & 1 \\
\hline 11035221 & 0 & 0 & 2 & 1 \\
\hline 11044881 & 0 & 0 & 2 & 1 \\
\hline 11045323 & 0 & 0 & 2 & 2 \\
\hline
\end{tabular}

(Continued) 
Table 5 (Continued)

\begin{tabular}{|c|c|c|c|c|}
\hline \multirow[t]{2}{*}{ Clinician ID } & \multicolumn{2}{|l|}{ Study eligible patients } & \multicolumn{2}{|c|}{ Study ineligible } \\
\hline & Total no. of patients & $\begin{array}{l}\text { No. of patients } \\
\text { statin prescribed }\end{array}$ & $\begin{array}{l}\text { Total no. of } \\
\text { patients }\end{array}$ & $\begin{array}{l}\text { No. of patients } \\
\text { statin prescribed }\end{array}$ \\
\hline 11046508 & 0 & 0 & 1 & 1 \\
\hline 11050138 & 0 & 0 & 1 & 1 \\
\hline 11051979 & 0 & 0 & 1 & 1 \\
\hline 12070 & 0 & 0 & 1 & 1 \\
\hline 12446 & 0 & 0 & 4 & 1 \\
\hline 12547 & 0 & 0 & 1 & 1 \\
\hline 12908 & 0 & 0 & 3 & 1 \\
\hline 14744 & 0 & 0 & 1 & 1 \\
\hline 14797 & 0 & 0 & 3 & 2 \\
\hline 17838 & 0 & 0 & 1 & 1 \\
\hline 17850 & 0 & 0 & 3 & 2 \\
\hline 18107 & 0 & 0 & 1 & 1 \\
\hline 18109 & 0 & 0 & 5 & 2 \\
\hline 18125 & 0 & 0 & 6 & 3 \\
\hline 18771 & 0 & 0 & 1 & 1 \\
\hline 18772 & 0 & 0 & 1 & 1 \\
\hline 19131 & 0 & 0 & 1 & 1 \\
\hline 20094 & 0 & 0 & 3 & 1 \\
\hline 20279 & 0 & 0 & 5 & 1 \\
\hline 20402 & 0 & 0 & 3 & 1 \\
\hline 20445 & 0 & 0 & 1 & 1 \\
\hline 20550 & 0 & 0 & 5 & 3 \\
\hline 20637 & 0 & 0 & 2 & 1 \\
\hline 21429 & 0 & 0 & 1 & 1 \\
\hline 21731 & 0 & 0 & 3 & 3 \\
\hline 21735 & 0 & 0 & 1 & 1 \\
\hline 22913 & 0 & 0 & 1 & 1 \\
\hline 22947 & 0 & 0 & 2 & 2 \\
\hline 23497 & 0 & 0 & 2 & 1 \\
\hline 23586 & 0 & 0 & 1 & 1 \\
\hline 23600 & 0 & 0 & 3 & 2 \\
\hline 23719 & 0 & 0 & 5 & 1 \\
\hline 23800 & 0 & 0 & 13 & 2 \\
\hline 23888 & 0 & 0 & 7 & 3 \\
\hline 24287 & 0 & 0 & 1 & 1 \\
\hline 24405 & 0 & 0 & 2 & 2 \\
\hline 24735 & 0 & 0 & 2 & 2 \\
\hline 24765 & 0 & 0 & 3 & 2 \\
\hline 24947 & 0 & 0 & 1 & 1 \\
\hline 25157 & 0 & 0 & 1 & 1 \\
\hline 25748 & 0 & 0 & 2 & 1 \\
\hline 25882 & 0 & 0 & 2 & 1 \\
\hline
\end{tabular}


Table 5 (Continued)

\begin{tabular}{|c|c|c|c|c|}
\hline \multirow[t]{2}{*}{ Clinician ID } & \multicolumn{2}{|l|}{ Study eligible patients } & \multicolumn{2}{|c|}{ Study ineligible } \\
\hline & Total no. of patients & $\begin{array}{l}\text { No. of patients } \\
\text { statin prescribed }\end{array}$ & $\begin{array}{l}\text { Total no. of } \\
\text { patients }\end{array}$ & $\begin{array}{l}\text { No. of patients } \\
\text { statin prescribed }\end{array}$ \\
\hline 26022 & 0 & 0 & 1 & 1 \\
\hline 26325 & 0 & 0 & 2 & 2 \\
\hline 26973 & 0 & 0 & 1 & 1 \\
\hline 27257 & 0 & 0 & 10 & 2 \\
\hline 27290 & 0 & 0 & 3 & 1 \\
\hline 27937 & 0 & 0 & 4 & 3 \\
\hline 28110 & 0 & 0 & 1 & 1 \\
\hline 28146 & 0 & 0 & 2 & 1 \\
\hline 28600 & 0 & 0 & 2 & 2 \\
\hline 28722 & 0 & 0 & 1 & 1 \\
\hline 28936 & 0 & 0 & 2 & 1 \\
\hline 29251 & 0 & 0 & 2 & 2 \\
\hline 29418 & 0 & 0 & 2 & 1 \\
\hline 29420 & 0 & 0 & 14 & 4 \\
\hline 29494 & 0 & 0 & 2 & 1 \\
\hline 29502 & 0 & 0 & 6 & 1 \\
\hline 29526 & 0 & 0 & 1 & 1 \\
\hline 29733 & 0 & 0 & 1 & 1 \\
\hline 29735 & 0 & 0 & 1 & 1 \\
\hline 29737 & 0 & 0 & 5 & 2 \\
\hline 29739 & 0 & 0 & 2 & 1 \\
\hline 29798 & 0 & 0 & 1 & 1 \\
\hline 29801 & 0 & 0 & 2 & 1 \\
\hline 29802 & 0 & 0 & 3 & 1 \\
\hline 29803 & 0 & 0 & 1 & 1 \\
\hline 29804 & 0 & 0 & 5 & 3 \\
\hline 29817 & 0 & 0 & 3 & 1 \\
\hline 29830 & 0 & 0 & 6 & 2 \\
\hline 29831 & 0 & 0 & 5 & 1 \\
\hline 30586 & 0 & 0 & 2 & 1 \\
\hline 30589 & 0 & 0 & 4 & 2 \\
\hline 30591 & 0 & 0 & 2 & 2 \\
\hline 30592 & 0 & 0 & 2 & 2 \\
\hline 30670 & 0 & 0 & 6 & 1 \\
\hline 30671 & 0 & 0 & 10 & 3 \\
\hline 30673 & 0 & 0 & 6 & 4 \\
\hline 30675 & 0 & 0 & 7 & 4 \\
\hline 30693 & 0 & 0 & 2 & 1 \\
\hline 30696 & 0 & 0 & 2 & 2 \\
\hline 31010 & 0 & 0 & 5 & 1 \\
\hline 31093 & 0 & 0 & 1 & 1 \\
\hline 31275 & 0 & 0 & 1 & 1 \\
\hline
\end{tabular}

(Continued) 
Table 5 (Continued)

\begin{tabular}{|c|c|c|c|c|}
\hline \multirow[t]{2}{*}{ Clinician ID } & \multicolumn{2}{|l|}{ Study eligible patients } & \multicolumn{2}{|c|}{ Study ineligible } \\
\hline & Total no. of patients & $\begin{array}{l}\text { No. of patients } \\
\text { statin prescribed }\end{array}$ & $\begin{array}{l}\text { Total no. of } \\
\text { patients }\end{array}$ & $\begin{array}{l}\text { No. of patients } \\
\text { statin prescribed }\end{array}$ \\
\hline 31541 & 0 & 0 & 1 & 1 \\
\hline 31544 & 0 & 0 & 1 & 1 \\
\hline 31557 & 0 & 0 & 8 & 3 \\
\hline 31558 & 0 & 0 & 6 & 3 \\
\hline 31562 & 0 & 0 & 6 & 2 \\
\hline 31567 & 0 & 0 & 6 & 3 \\
\hline 31575 & 0 & 0 & 14 & 6 \\
\hline 31576 & 0 & 0 & 4 & 1 \\
\hline 31587 & 0 & 0 & 3 & 1 \\
\hline 31597 & 0 & 0 & 6 & 1 \\
\hline 31599 & 0 & 0 & 1 & 1 \\
\hline 32450 & 0 & 0 & 1 & 1 \\
\hline 32464 & 0 & 0 & 1 & 1 \\
\hline 32466 & 0 & 0 & 1 & 1 \\
\hline 32470 & 0 & 0 & 1 & 1 \\
\hline 32485 & 0 & 0 & 5 & 3 \\
\hline 32490 & 0 & 0 & 3 & 2 \\
\hline 32491 & 0 & 0 & 5 & 4 \\
\hline 32493 & 0 & 0 & 4 & 4 \\
\hline 32494 & 0 & 0 & 3 & 2 \\
\hline 32498 & 0 & 0 & 1 & 1 \\
\hline 32500 & 0 & 0 & 1 & 1 \\
\hline 33011 & 0 & 0 & 4 & 2 \\
\hline 3722 & 0 & 0 & 1 & 1 \\
\hline 4050 & 0 & 0 & 1 & 1 \\
\hline 505804 & 0 & 0 & 1 & 1 \\
\hline 5279 & 0 & 0 & 1 & 1 \\
\hline 5512 & 0 & 0 & 4 & 2 \\
\hline 5777 & 0 & 0 & 1 & 1 \\
\hline 6319 & 0 & 0 & 5 & 1 \\
\hline 6845 & 0 & 0 & 3 & 3 \\
\hline 7231 & 0 & 0 & 1 & 1 \\
\hline 7421 & 0 & 0 & 1 & 1 \\
\hline 9782 & 0 & 0 & 1 & 1 \\
\hline
\end{tabular}

Abbreviation: ID, identification.

Most clinicians were internal medicine and family medicine physicians. Other specialties were represented because the primary care clinician listed in the EHR can be from any specialty. As our study was performed at a tertiary referral center, neurologists and surgeons may be the EHR-listed primary care clinician.

The statin macro is a clinician-initiated CDS tool. Our results may be confounded as clinicians who used the statin macro may be more technologically proficient or more compliant to statin guidelines. However, almost all the 125 clinicians who used the statin macro for patients also did not use the statin macro for other patients. Therefore, our results are not due to a small number of clinicians who were the only users of the statin macro.

The statin macro was used for a low percentage (3.2\%) of patients recommended but not prescribed statin therapy. 
Table 6 Multivariate adjusted mixed effect logistic regression model for statin therapy prescription during study period

\begin{tabular}{|l|l|l|}
\hline & ${\text { Odds ratio }(95 \% \mathrm{Cl})^{\mathrm{a}}}$ & p-Value $^{\mathrm{b}}$ \\
\hline Statin macro usage & $2.86(2.24-3.65)$ & $<\mathbf{0 . 0 0 1}$ \\
\hline Male & $1.19(1.04-1.36)$ & 0.011 \\
\hline Age & $\mathbf{1 . 0 3 ( 1 . 0 2 - 1 . 0 4 )}$ & $<\mathbf{0 . 0 0 1}$ \\
\hline Black & $0.88(0.70-1.09)$ & 0.22 \\
\hline Smoke & $1.25(1.00-1.55)$ & 0.054 \\
\hline DM & $2.61(2.26-3.02)$ & $<\mathbf{0 . 0 0 1}$ \\
\hline Antihypertensive & $\mathbf{1 . 3 2 ( 1 . 1 7 - 1 . 4 9 )}$ & $<\mathbf{0 . 0 0 1}$ \\
\hline Systolic BP & $1.00(1.00-1.00)$ & 0.62 \\
\hline Total cholesterol & $\mathbf{1 . 0 1 ( 1 . 0 0 - 1 . 0 1 )}$ & $<\mathbf{0 . 0 0 1}$ \\
\hline LDL & $1.00(1.00-1.00)$ & 0.25 \\
\hline HDL & $\mathbf{0 . 9 8}(0.98-0.99)$ & $<\mathbf{0 . 0 0 1}$ \\
\hline
\end{tabular}

Abbreviations: BP, blood pressure; $\mathrm{Cl}$, confidence interval; DM, diabetes mellitus; HDL, high-density lipoprotein; LDL, low-density lipoprotein. Note: Bold indicates variables with significant $p$-values.

${ }^{a}$ Odds ratio of variables from mixed effect logistic regression.

${ }^{\mathrm{b}} \mathrm{p}$-Value based on likelihood ratio test while controlling for other variables in the model. Clinician was included as a random effect.

Other studies of clinician-initiated CDS tools showed very low uptake, which was $0 \%$ during some months or at some clinical sites. ${ }^{39,40}$ Aside from low advertisement, the statin macro may have had low uptake as not all primary care visits were focused on prevention. Some visits may have focused on acute issues. CDS features associated with low uptake included clinician perception of loss of autonomy, lack of EHR integration, poor transparency of CDS developers, lack of clinical leadership endorsement, lack of financial incentive, and changing guidelines. ${ }^{41,42}$ Ongoing systematic reviews will further delineate these features. ${ }^{43}$ Future strategies to improve statin macro uptake include advertisements describing developers, emphasizing clinical leadership endorsement, and financial incentives.

There are many reasons that clinicians may not prescribe statin therapy according to the 2013 ACC/AHA guideline. In 2015 , less than half of surveyed clinicians read the guideline, knew the patient groups recommended statin therapy, or knew the definition of statin intensity. ${ }^{44}$ The 2013 ACC/AHA statin guideline was met with controversy as there were no LDL target goals and the number of patients recommended statin therapy would significantly increase compared with previous guidelines. ${ }^{45,46}$ There was also concern that the risk equation overestimated the 10-year ASCVD risk. ${ }^{47}$ The 2013 ACC/AHA guideline considered only LDL-C rather than other lipoprotein measures such as LDL particle number or lipoprotein (a), which were associated with cardiovascular risk. ${ }^{48,49}$ Of clinicians who were knowledgeable about the 2013 ACC/AHA statin guidelines, many disagreed with statin intensity definitions and the groups' recommended statin therapy. ${ }^{50}$

Given the benefits of the statin macro, one could consider developing a system-initiated statin CDS tool. Previous studies showed system-initiated CDS tools improved clinical outcomes compared with clinician-initiated CDS tools. ${ }^{18}$ However, system-initiated CDS tools, such as best practice alerts, may lead to alert fatigue. ${ }^{51,52}$ A future randomized control trial comparing clinician-initiated versus systeminitiated statin CDS tools could compare this CDS feature and provide a greater certainty of the statin CDS tool effect size.

There were limitations to this study. We calculated the 2013 ACC/AHA statin guideline recommendation for all patients before the study period as of December 31, 2015. We could not determine the statin recommendation for each patient at the time of statin macro usage or if the statin macro was used in a template versus on demand due to limitations of EHR data archiving. We did not determine if each patient's statin prescription met the ACC/AHA 2013 therapy intensity recommendations (high vs. moderate vs. low). We did not determine if clinicians using the statin macro tool initiated its use because they already decided to prescribe a statin; if so, interpretation of study results may be affected. We plan to collect time-specific patient data for future studies with macros and could implement CDS monitoring. ${ }^{53}$ Since initiation of the study, a new 2018 ACC/AHA statin guideline was recently published. ${ }^{54}$ Future versions of the statin macro should include updated 2018 ACC/AHA statin guidelines.

\section{Conclusion}

Statin macro usage was associated with improved 2013 ACC/ AHA statin guideline compliance at the level of statin prescription versus no statin prescription. We did not determine if each patient's statin prescription met the ACC/AHA 2013 therapy intensity recommendations (high vs. moderate vs. low). Macro CDS tools may improve compliance to other societal guidelines.

\section{Clinical Relevance Statement}

Although many best practice guidelines exist for initiating medication in select patient groups, these medications are prescribed at suboptimal rates. Clinical decision support tools may improve guideline compliance. Use of a statin macro was associated with improved statin guideline compliance.

\section{Multiple Choice Questions}

1. The following baseline characteristics were significantly different in patients for whom the statin macro was and was not used:

a. High-density lipoprotein and race.

b. Systolic blood pressure and total cholesterol.

c. Race and systolic blood pressure.

d. Gender and age.

e. Diabetes mellitus and low-density lipoprotein.

Correct Answer: The correct answer is option e. Baseline characteristics including diabetes mellitus and LDL were 
significantly different in patients for whom the statin macro was and was not used. However, the large sample size lead to statistical significance with somewhat trivial clinically significant differences.

2. The following variables were significantly associated with statin prescription in patients who were recommended a statin based on guidelines but not previously prescribed a statin:

a. Statin macro usage, diabetes mellitus.

b. Race, diabetes mellitus.

c. Statin macro usage, race.

d. Statin macro usage, systolic blood pressure.

Correct Answer: The correct answer is option a. Statin macro usage and diabetes mellitus were significantly associated with statin prescription while controlling for other covariates.

\section{Protection of Human and Animal Subjects}

The University of California, Los Angeles Institutional Review Board approved a waiver of authorization for this study (IRB\#: 16-001676).

\section{Funding}

This research was supported by NIH National Center for Advancing Translational Science (NCATS) UCLA CTSI Grant Number UL1TR001881.

\section{Conflict of Interest}

None declared.

\section{Acknowledgments}

We thank the UCLA Health System, its clinicians, and the patients.

\section{References}

1 Grol R, Grimshaw J. From best evidence to best practice: effective implementation of change in patients' care. Lancet 2003;362 (9391):1225-1230

2 Stone NJ, Robinson JG, Lichtenstein AH, et al; American College of Cardiology/American Heart Association Task Force on Practice Guidelines. 2013 ACC/AHA guideline on the treatment of blood cholesterol to reduce atherosclerotic cardiovascular risk in adults: a report of the American College of Cardiology/American Heart Association Task Force on Practice Guidelines. Circulation 2014;129(25, Suppl 2):S1-S45

3 Housholder-Hughes SD, Martin MM, McFarland MR, Creech CJ, Shea MJ. Healthcare provider compliance with the 2013 ACC/AHA Adult Cholesterol Guideline recommendation for high-intensity dose statins for patients with coronary artery disease. Heart Lung 2017;46(04):328-333

4 Bavishi A, Howard T, Kim JP, et al. Treatment gap in primary prevention patients presenting with acute coronary syndrome. Am J Cardiol 2019;123(03):368-374

5 Fischer F, Lange K, Klose K, Greiner W, Kraemer A. Barriers and strategies in guideline implementation-a scoping review. Healthcare (Basel) 2016;4(03):36

6 Carthey J, Walker S, Deelchand V, Vincent C, Griffiths WH. Breaking the rules: understanding non-compliance with policies and guidelines. BMJ 2011;343:d5283

7 National Cholesterol Education Program (NCEP) Expert Panel on Detection, Evaluation, and Treatment of High Blood Cholesterol in
Adults (Adult Treatment Panel III). Third Report of the National Cholesterol Education Program (NCEP) Expert Panel on Detection, Evaluation, and Treatment of High Blood Cholesterol in Adults (Adult Treatment Panel III) final report. Circulation 2002;106(25): 3143-3421

8 D'Agostino RB Sr, Vasan RS, Pencina MJ, et al. General cardiovascular risk profile for use in primary care: the Framingham Heart Study. Circulation 2008;117(06):743-753

9 Hippisley-Cox J, Coupland C, Vinogradova Y, Robson J, May M, Brindle P. Derivation and validation of QRISK, a new cardiovascular disease risk score for the United Kingdom: prospective open cohort study. BMJ 2007;335(7611):136

10 American College of Cardiology. ASCVD Risk Estimator. 2013. Available at: http://tools.cardiosource.org/ASCVD-Risk-Estimator/. Accessed December 1, 2013

11 American College of Cardiology. ASCVD Risk Calculator. 2013. Available at: https://professional.heart.org/professional/GuidelinesStatements/ASCVDRiskCalculator/UCM_457698_ASCVD-Risk -Calculator.jsp. Accessed December 1, 2013

12 North F, Fox S, Chaudhry R. Clinician time used for decision making: a best case workflow study using cardiovascular risk assessments and Ask Mayo Expert algorithmic care process models. BMC Med Inform Decis Mak 2016;16:96

13 Fretheim A, Flottorp S, Oxman A. Effect of interventions for implementing clinical practice guidelines. Oslo, Norway: The Norwegian Institute of Public Health (NIPH); 2015

14 Sequist TD, Gandhi TK, Karson AS, et al. A randomized trial of electronic clinical reminders to improve quality of care for diabetes and coronary artery disease. J Am Med Inform Assoc 2005;12(04):431-437

15 Smith MW, Brown C, Virani SS, et al. Incorporating guideline adherence and practice implementation issues into the design of decision support for beta-blocker titration for heart failure. Appl Clin Inform 2018;9(02):478-489

16 Jones BE, Collingridge DS, Vines CG, et al. CDS in a learning health care system: identifying physicians' reasons for rejection of best-practice recommendations in pneumonia through computerized clinical decision support. Appl Clin Inform 2019;10 (01):1-9

17 Au L, Oster A, Yeh GH, Magno J, Paek HM. Utilizing an electronic health record system to improve vaccination coverage in children. Appl Clin Inform 2010;1(03):221-231

18 Van de Velde S, Heselmans A, Delvaux N, et al. A systematic review of trials evaluating success factors of interventions with computerised clinical decision support. Implement Sci 2018;13 (01):114

19 Roshanov PS, Fernandes N, Wilczynski JM, et al. Features of effective computerised clinical decision support systems: metaregression of 162 randomised trials. BMJ 2013;346:f657

20 Aspry KE, Furman R, Karalis DG, et al. Effect of health information technology interventions on lipid management in clinical practice: a systematic review of randomized controlled trials. J Clin Lipidol 2013;7(06):546-560

21 Scheitel MR, Kessler ME, Shellum JL, et al. Effect of a novel clinical decision support tool on the efficiency and accuracy of treatment recommendations for cholesterol management. Appl Clin Inform 2017;8(01):124-136

22 Bright TJ, Wong A, Dhurjati R, et al. Effect of clinical decisionsupport systems: a systematic review. Ann Intern Med 2012;157 (01):29-43

23 Epic Systems. Epic. Verona, WI: Epic Systems; 2016

24 Rattay KT, Ramakrishnan M, Atkinson A, Gilson M, Drayton V. Use of an electronic medical record system to support primary care recommendations to prevent, identify, and manage childhood obesity. Pediatrics 2009;123(Suppl 2):S100-S107

25 Young A. 'SWINEUPDATE': using EMR charting tools as a clinical decision support tool during the H1N1 outbreak. WMJ 2010;109 (04):222-223 
26 O'Connor PJ, Sperl-Hillen JM, Rush WA, et al. Impact of electronic health record clinical decision support on diabetes care: a randomized trial. Ann Fam Med 2011;9(01):12-21

27 Tierney WM, Overhage JM, Murray MD, et al. Effects of computerized guidelines for managing heart disease in primary care. J Gen Intern Med 2003;18(12):967-976

28 van Wyk JT, van Wijk MAM, Sturkenboom MCJM, Mosseveld M, Moorman PW, van der Lei J. Electronic alerts versus on-demand decision support to improve dyslipidemia treatment: a cluster randomized controlled trial. Circulation 2008;117(03):371-378

29 Lester WT, Grant RW, Barnett GO, Chueh HC. Randomized controlled trial of an informatics-based intervention to increase statin prescription for secondary prevention of coronary disease. J Gen Intern Med 2006;21(01):22-29

30 Van de Velde S, Kunnamo I, Roshanov P, et al; GUIDES expert panel. The GUIDES checklist: development of a tool to improve the successful use of guideline-based computerised clinical decision support. Implement Sci 2018;13(01):86

31 Weiskopf NG, Weng C. Methods and dimensions of electronic health record data quality assessment: enabling reuse for clinical research. J Am Med Inform Assoc 2013;20(01):144-151

32 Johnson SG, Speedie S, Simon G, Kumar V, Westra BL. Application of an ontology for characterizing data quality for a secondary use of EHR data. Appl Clin Inform 2016;7(01):69-88

33 Bates D, Mächler M, Bolker B, et al. Fitting linear mixed-effects models using lme4. J Stat Softw 2015;67:1-48

34 Rubin DB. Statistical matching using file concatenation with adjusted weights and multiple imputations. J Bus Econ Stat 1986; 4:87-94

35 Rubin DB. Multiple Imputation for Nonresponse in Surveys. New York: John Wiley and Sons; 1987

36 Van Buuren S. Flexible Imputation of Missing Data. Boca Raton, FL: Chapman and Hall/CRC; 2018

37 Harrell FE Jr. Hmisc: Harrell Miscellaneous. Nashville, TN: Vanderbilt University; 2019

38 R Core Team. R: A Language and Environment for Statistical Computing. Vienna, Austria: R Foundation for Statistical Computing; 2018

39 Eccles M, McColl E, Steen N, et al. Effect of computerised evidence based guidelines on management of asthma and angina in adults in primary care: cluster randomised controlled trial. BMJ 2002; 325(7370):941

40 Hobbs FD, Delaney BC, Carson A, Kenkre JE. A prospective controlled trial of computerized decision support for lipid management in primary care. Fam Pract 1996;13(02):133-137

41 Liberati EG, Ruggiero F, Galuppo L, et al. What hinders the uptake of computerized decision support systems in hospitals? A qualitative study and framework for implementation. Implement Sci 2017;12(01):113
42 Moxey A, Robertson J, Newby D, Hains I, Williamson M, Pearson SA. Computerized clinical decision support for prescribing: provision does not guarantee uptake. J Am Med Inform Assoc 2010;17 (01):25-33

43 Kouri A, Yamada J, Gupta S. Identifying factors related to user uptake of computerized clinical decision support systems: a systematic review and meta-regression. PROSPERO 2018: CRD42018092337. Available at: http://www.crd.york.ac.uk/ PROSPERO/display_record.php?ID=CRD42018092337. Accessed May 17, 2019

44 Virani SS, Pokharel Y, Steinberg L, et al. Provider understanding of the 2013 ACC/AHA cholesterol guideline. J Clin Lipidol 2016;10 (03):497-504.e4

45 Ridker PM, Cook NR. Statins: new American guidelines for prevention of cardiovascular disease. Lancet 2013;382(9907): 1762-1765

46 Pencina MJ, Navar-Boggan AM, D’Agostino RB Sr, et al. Application of new cholesterol guidelines to a population-based sample. $\mathrm{N}$ Engl J Med 2014;370(15):1422-1431

47 Amin NP, Martin SS, Blaha MJ, Nasir K, Blumenthal RS, Michos ED. Headed in the right direction but at risk for miscalculation: a critical appraisal of the 2013 ACC/AHA risk assessment guidelines. J Am Coll Cardiol 2014;63(25 Pt A):2789-2794

48 Otvos JD, Mora S, Shalaurova I, Greenland P, Mackey RH, Goff DC Jr. Clinical implications of discordance between low-density lipoprotein cholesterol and particle number. J Clin Lipidol 2011;5(02):105-113

49 Erqou S, Kaptoge S, Perry PL, et al; Emerging Risk Factors Collaboration. Lipoprotein(a) concentration and the risk of coronary heart disease, stroke, and nonvascular mortality. JAMA 2009;302(04):412-423

50 Setia S, Fung SS-W, Waters DD. Doctors' knowledge, attitudes, and compliance with 2013 ACC/AHA guidelines for prevention of atherosclerotic cardiovascular disease in Singapore. Vasc Health Risk Manag 2015;11:303-310

51 Rehr CA, Wong A, Seger DL, Bates DW. Determining inappropriate medication alerts from "inaccurate warning" overrides in the intensive care unit. Appl Clin Inform 2018;9(02):268-274

52 Ancker JS, Edwards A, Nosal S, Hauser D, Mauer E, Kaushal R; with the HITEC Investigators. Effects of workload, work complexity, and repeated alerts on alert fatigue in a clinical decision support system. BMC Med Inform Decis Mak 2017;17(01):36

53 Yoshida E, Fei S, Bavuso K, Lagor C, Maviglia S. The value of monitoring clinical decision support interventions. Appl Clin Inform 2018;9(01):163-173

54 Grundy SM, Stone NJ, Bailey AL, et al. AHA/ACC/AACVPR/AAPA/ ABC/ACPM/ADA/AGS/APhA/ASPC/NLA/PCNA guideline on the management of blood cholesterol: a report of the American College of Cardiology/American Heart Association Task Force on Clinical Practice Guidelines. J Am Coll Cardiol 2018;2018:25709 